TRANSACTIONS OF THE

AMERICAN MATHEMATICAL SOCIETY

Volume 355, Number 10, Pages 4061-4091

S 0002-9947(03)03338-

Article electronically published on June 24, 2003

\title{
TRIBASIC INTEGRALS AND IDENTITIES OF ROGERS-RAMANUJAN TYPE
}

\author{
M. E. H. ISMAIL AND D. STANTON
}

\begin{abstract}
Some integrals involving three bases are evaluated as infinite products using complex analysis. Many special cases of these integrals may be evaluated in another way to find infinite sum representations for these infinite products. The resulting identities are identities of Rogers-Ramanujan type. Some integer partition interpretations of these identities are given. Generalizations of the Rogers-Ramanujan type identities involving polynomials are given again as corollaries of integral evaluations.
\end{abstract}

\section{INTRODUCTION}

The purpose of this paper is to show that integral evaluations involving infinite products with three independent bases lead to identities of Rogers-Ramanujan type.

In [10] it was shown that the Rogers-Ramanujan identities of modulus 5 follow from evaluating the bibasic integral

$$
\frac{\left(q t^{2} ; q\right)_{\infty}}{2 \pi} \int_{0}^{\pi} \frac{\left(q^{5}, e^{2 i \theta}, e^{-2 i \theta} ; q^{5}\right)_{\infty}}{\left(t e^{i \theta}, t e^{-i \theta} ; q\right)_{\infty}} d \theta
$$

We generalize the above integral by replacing the three bases of the infinite products $q^{5}, q$ and $q$ by independent bases $s, p$ and $q$. The resulting integral can be evaluated as an infinite product for special values of $t$. By specializing $s, p$, and $q$, the product sides of identities of Rogers-Ramanujan type appear. The special values of $t$ for which such identities exist can be found by considering the singularities of the integrals as functions of $t$.

To find the sum sides of these identities of Rogers-Ramanujan type, we use techniques from classical orthogonal polynomials. Once $s$ has been specialized, we realize that evaluating these integrals is equivalent to a certain connection coefficient problem between orthogonal polynomials which are basic hypergeometric series with arbitrary bases $p$ and $q$. The weight function and generating function for the $q$-Hermite polynomials motivates our choices of integrals. Judicious choices of the base $p$ will allow for an explicit solution of the connection coefficient problem and thus a different evaluation of the integral as a sum.

The tribasic integrals are given in Theorems 2, 3, 4 and 5 of $\S 2$. The details of the orthogonal polynomial method are described in $\S 3$. It is applied to the $q$ Hermite polynomials in $\S 4$, and another set of polynomials is similarly considered

Received by the editors June 16, 2002.

2000 Mathematics Subject Classification. Primary 33D45, 10J20; Secondary 33E20.

This research was partially supported by NSF grants DMS 99-70865, DMS 99-70627, and the Liu Bie Ju Center for Mathematical Sciences. 
in $\S 5$. The four classes of integrals we evaluate are given in (4.1), (4.2), (4.3), and (5.1). The often lengthy proofs of computational lemmas in $\S 4$ and $\S 5$ are collected in an Appendix, $\S 9$. $\S 6$ contains a double series identity of Rogers-Ramanujan type. It is this master identity that contains all the special cases that lead to the single sum identities of $\S 5$. Finally, the multisum identities are developed in $\S 7$.

In addition, we identify polynomials that correspond to the polynomials $\left\{a_{n}(q)\right\}$ and $\left\{b_{n}(q)\right\}$ introduced by Schur 14 in his proof of the Rogers-Ramanujan identities. In particular, we extend some of the results in 10 to moduli other than 5 . $\S 8$ contains remarks about certain aspects of our work. Combinatorial interpretations and positivity results are found throughout the paper.

We will use the standard notation for $q$-series and infinite products found in [5. and [11.

We use the Jacobi triple identity

$$
(x, q / x, q ; q)_{\infty}=\sum_{n=-\infty}^{\infty}(-1)^{n} q^{\left(\begin{array}{c}
n \\
2
\end{array}\right)} x^{n},
$$

and the quintuple product identity

$$
\begin{array}{r}
(q, 1 / x, q x, q ; q)_{\infty}\left(q x^{2}, q / x^{2} ; q^{2}\right)_{\infty}=\sum_{m=-\infty}^{\infty} x^{3 m} q^{\left(3 m^{2}+m\right) / 2}\left(1-q^{-m} / x\right) \\
=\left(q^{3},-q^{2} x^{3},-q / x^{3} ; q^{3}\right)_{\infty}-\left(q^{3},-q x^{3},-q^{2} / x^{3} ; q^{3}\right)_{\infty} / x
\end{array}
$$

We will also use methods from complex analysis in $\S 2$.

\section{SCHWARZ'S THEOREM AND INTEGRAL EVALUATIONS}

In this section we give, in Theorems 2 and 3, integral evaluations each involving three independent bases $p, q$, and $s$. These evaluations follow from Schwarz's theorem for the Poisson kernel; the relevant variation is stated in Theorem 1. By specializing one of the bases, the integral of Theorem 3 may be analytically continued as a function of $t$. The explicit bibasic evaluations are given in Theorems 4 and 5 .

Schwarz's theorem [1, $(\S 6.4)]$ asserts that if $f(\theta)$ is piecewise continuous on $[0,2 \pi]$, then

$$
\lim _{z \rightarrow r e^{i \phi}} \int_{0}^{2 \pi} \frac{r^{2}-|z|^{2}}{2 \pi\left|r e^{i \theta}-z\right|^{2}} f(\theta) d \theta=f(\phi),
$$

provided that $f$ is continuous at $\phi$. We shall use a minor modification of Schwarz's result.

Theorem 1. Let $f(\theta, z)$ be continuous in $\theta$ for $\theta \in[0,2 \pi]$, and for all $z$ so that $r \geq|z| \geq r-\epsilon$ for some positive $\epsilon$. Assume further that $f(\theta, z)$ converges to $f\left(\theta, r e^{i \phi}\right)$ as $z \rightarrow r e^{i \phi}$ uniformly in $\theta$, for $\theta \in[0,2 \pi]$. Then

$$
\lim _{z \rightarrow r e^{i \phi}} \int_{0}^{2 \pi} \frac{\left(r^{2}-|z|^{2}\right) f(\theta, z) d \theta}{2 \pi\left|r e^{i \theta}-z\right|^{2}}=f\left(\phi, r e^{i \phi}\right) .
$$


Proof. Since $\int_{0}^{2 \pi}\left[r^{2}-|z|^{2}\right] d \theta /\left[\left|r e^{i \theta}-z\right|^{2}\right]=2 \pi$, we obtain

$$
\begin{gathered}
\left|\int_{0}^{2 \pi} \frac{r^{2}-|z|^{2}}{2 \pi\left|r e^{i \theta}-z\right|^{2}} f(\theta, z) d \theta-f\left(\phi, r e^{i \phi}\right)\right| \\
=\left|\int_{0}^{2 \pi} \frac{r^{2}-|z|^{2}}{2 \pi\left|r e^{i \theta}-z\right|^{2}}\left[f(\theta, z)-f\left(\theta, r e^{i \phi}\right)+f\left(\theta, r e^{i \phi}\right)-f\left(\phi, r e^{i \phi}\right)\right] d \theta\right| \\
\leq \sup _{\theta \in[0,2 \pi]}\left|f(\theta, z)-f\left(\theta, r e^{i \phi}\right)\right|+\left|\int_{0}^{2 \pi} \frac{r^{2}-|z|^{2}}{2 \pi\left|r e^{i \theta}-z\right|^{2}} f\left(\theta, r e^{i \phi}\right) d \theta-f\left(\phi, r e^{i \phi}\right)\right| .
\end{gathered}
$$

The first term tends to zero as $z \rightarrow r e^{i \phi}$ by the uniform convergence hypothesis, while the second term tends to zero from Schwarz's theorem.

We next apply Theorem 1 to find limiting values of the tribasic integrals

$$
G_{1}(t, p, q, s)=\frac{(-t ; q)_{\infty}}{2 \pi} \int_{0}^{\pi} \frac{\left(s, e^{2 i \theta}, e^{-2 i \theta} ; s\right)_{\infty}}{\left(t e^{2 i \theta}, t e^{-2 i \theta} ; p\right)_{\infty}} d \theta, \quad|t|<1 .
$$

Theorem 2. We have

$$
\lim _{t \rightarrow-1} G_{1}(t, p, q, s)=\frac{(q ; q)_{\infty}}{(p, p ; p)_{\infty}}(s,-s,-s ; s)_{\infty} .
$$

Proof. Let

$$
F(z)=\left(s, z^{2}, 1 / z^{2} ; s\right)_{\infty}
$$

Clearly,

$$
\begin{aligned}
\lim _{t \rightarrow-1} G_{1}(t, p, q, s) & =\lim _{t \rightarrow-1} \frac{(-t ; q)_{\infty}}{4 \pi} \int_{0}^{2 \pi} \frac{F\left(e^{i \theta / 2}\right) d \theta}{\left(t e^{i \theta}, t e^{-i \theta} ; p\right)_{\infty}} \\
& =\frac{(q ; q)_{\infty}}{8 \pi} \lim _{t \rightarrow-1} \int_{0}^{2 \pi} \frac{\left(1-t^{2}\right) F\left(e^{i \theta / 2}\right) d \theta}{\left|e^{i \theta}-t\right|^{2}\left(t p e^{i \theta}, t p e^{-i \theta} ; p\right)_{\infty}} \\
& =\frac{1}{4} \frac{(q ; q)_{\infty} F\left(e^{i \pi / 2}\right)}{\left(-p e^{i \pi},-p e^{-i \pi} ; p\right)_{\infty}} \\
& =\frac{(q ; q)_{\infty}}{(p, p ; p)_{\infty}}(s,-s,-s ; s)_{\infty},
\end{aligned}
$$

by applying Theorem 1 with $\phi=\pi, r=1$, and

$$
f(\theta, z)=F\left(e^{i \theta / 2}\right) /\left(z p e^{i \theta}, z p e^{-i \theta} ; p\right)_{\infty} .
$$

Another tribasic integral is defined by

$$
G_{2}(t, p, q, s)=\frac{\left(p t^{2} ; q\right)_{\infty}}{2 \pi} \int_{0}^{\pi} \frac{\left(s, e^{2 i \theta}, e^{-2 i \theta} ; s\right)_{\infty}}{\left(t e^{i \theta}, t e^{-i \theta} ; p\right)_{\infty}} d \theta, \quad|t|<1 .
$$

We next consider an analytic continuation and a limiting behavior of $G_{2}$.

Theorem 3. The function $G_{2}(t, p, q, s)$ can be analytically continued to the disc $|t|<p^{-1 / 2}$. The analytic continuation to $p^{1 / 2}<|t|<p^{-1 / 2}$ is given by

$$
\begin{aligned}
G_{2}(t, p, q, s)= & \frac{\left(p t^{2} ; q\right)_{\infty}}{4 \pi} \int_{0}^{2 \pi} \frac{\left(s, e^{2 i \theta} / p, p e^{-2 i \theta} ; s\right)_{\infty} d \theta}{\left(t p^{-1 / 2} e^{i \theta}, t p^{1 / 2} e^{-i \theta} ; p\right)_{\infty}} \\
& +\frac{\left(s, 1 / t^{2}, s t^{2} ; s\right)_{\infty}\left(p t^{2} ; q\right)_{\infty}}{2\left(p, p t^{2} ; p\right)_{\infty}} .
\end{aligned}
$$


Furthermore,

$$
\lim _{t \rightarrow p^{-1 / 2}} G_{2}(t, p, q, s)=\frac{(s, p, s / p ; s)_{\infty}(q ; q)_{\infty}}{(p, p ; p)_{\infty}} .
$$

Proof. First we analytically continue $G_{2}(t, p, q, s)$ to the annulus $|t| \in\left(p^{1 / 2}, p^{-1 / 2}\right)$. Note that for $p^{1 / 2}<t<1$ we have

$$
\begin{aligned}
G_{2}(t, p, q, s) & =\frac{\left(p t^{2} ; q\right)_{\infty}}{4 \pi} \int_{-\pi}^{\pi} \frac{\left(s, e^{2 i \theta}, e^{-2 i \theta} ; s\right)_{\infty}}{\left(t e^{i \theta}, t e^{-i \theta} ; p\right)_{\infty}} d \theta \\
& =\frac{\left(p t^{2} ; q\right)_{\infty}}{4 \pi} \int_{|z|=1} \frac{F(z)}{(t z, t / z ; p)_{\infty}} \frac{d z}{i z} \\
& =\frac{\left(p t^{2} ; q\right)_{\infty}}{4 \pi} \int_{|z|=p^{-1 / 2}} \frac{F(z)}{(t z, t / z ; p)_{\infty}} \frac{d z}{i z}-R
\end{aligned}
$$

where $R$ is the sum of residues at the poles in the domain $1<|z|<p^{-1 / 2}$. The only pole is at $z=1 / t$; thus,

$$
R=-\frac{\left(s, 1 / t^{2}, t^{2} ; s\right)_{\infty}\left(p t^{2} ; q\right)_{\infty}}{2\left(p, t^{2} ; p\right)_{\infty}}
$$

and

$$
\begin{gathered}
G_{2}(t, p, q, s)=\frac{\left(p t^{2} ; q\right)_{\infty}}{4 \pi} \int_{0}^{2 \pi} \frac{F\left(p^{-1 / 2} e^{i \theta}\right)}{\left(t p^{-1 / 2} e^{i \theta}, t p^{1 / 2} e^{-i \theta} ; p\right)_{\infty}} d \theta \\
+\frac{\left(s, 1 / t^{2}, s t^{2} ; s\right)_{\infty}\left(p t^{2} ; q\right)_{\infty}}{2\left(p, p t^{2} ; p\right)_{\infty}}
\end{gathered}
$$

which is Theorem 3. The right-hand side of $G_{2}(t, p, q, s)$ is clearly analytic in $t$ for $p^{1 / 2}<|t|<p^{-1 / 2}$. The limiting case follows from Theorem 1 with $\phi=0$, $r=p^{-1 / 2}$, and

$$
f(\theta, z)=F\left(p^{-1 / 2} e^{i \theta}\right) /\left(1-t p^{-1 / 2} e^{i \theta}\right)\left(z p^{3 / 2} e^{i \theta}, z p^{3 / 2} e^{-i \theta} ; p\right)_{\infty} .
$$

The next result gives an analytic continuation of $G_{2}(t, p, q, s)$ valid for $1<|t|<$ $1 / p$, but to do so we have to choose $q=p$.

Theorem 4. An analytic continuation of $G_{2}(t, p, p, s)$ to $1<|t|<1 / p$ may be given by

$$
\begin{aligned}
G_{2}(t, p, p, s)= & \frac{\left(p t^{2} ; p\right)_{\infty}}{4 \pi} \int_{0}^{2 \pi} \frac{F\left(e^{i \theta} / p\right)}{\left(t e^{i \theta} / p, t p e^{-i \theta} ; p\right)_{\infty}} d \theta \\
& +\frac{\left(s, 1 / t^{2}, s t^{2} ; s\right)_{\infty}}{2(p ; p)_{\infty}}-\frac{p\left(s, p^{2} t^{2}, 1 / p^{2} t^{2} ; s\right)_{\infty}}{2(1-p)(p ; p)_{\infty}} .
\end{aligned}
$$

Furthermore,

$$
\lim _{t \rightarrow 1 / p} G_{2}(t, p, p, s)=\frac{\left(s, p^{2}, s / p^{2} ; s\right)_{\infty}}{(p ; p)_{\infty}} .
$$

Proof. Theorem 3 gives an analytic continuation of $G_{2}(t, p, p, s)$ to $p^{1 / 2}<|t|<$ $p^{-1 / 2}$. Therefore, for $1<|t|<p^{-1 / 2}$, we have

$$
G_{2}(t, p, p, s)-\frac{\left(s, 1 / t^{2}, s t^{2} ; s\right)_{\infty}}{2(p ; p)_{\infty}}=\frac{\left(p t^{2} ; p\right)_{\infty}}{4 \pi} \int_{|z|=p^{-1 / 2}} \frac{F\left(p^{-1 / 2} z\right)}{\left(t p^{-1 / 2} z, t p^{1 / 2} / z ; p\right)_{\infty}} \frac{d z}{i z}-R
$$


and $R$ is the contribution from the poles in $1<|z|<p^{-1 / 2}$. Again, the only pole in this annulus is when $z t p^{1 / 2}=1$. Thus

$$
R=\frac{p\left(s, p^{2} t^{2}, 1 / p^{2} t^{2} ; s\right)_{\infty}}{2(1-p)(p ; p)_{\infty}}
$$

and Theorem 4 follows. The limiting case follows from Theorem 1.

We now generalize Theorem 4 by analytically continuing $G_{2}(t, p, p, s)$ to annuli of the type $p^{1-k / 2}<|t|<p^{-k / 2}$.

Theorem 5. For $k=1,2, \ldots, G_{2}(t, p, p, s)$ can be continued to $p^{1-k / 2}<|t|<$ $p^{-k / 2}$ through

$$
\begin{gathered}
G_{2}(t, p, p, s)=\frac{\left(p t^{2} ; p\right)_{\infty}}{4 \pi} \int_{0}^{2 \pi} \frac{F\left(e^{i \theta} p^{-k / 2}\right)}{\left(t e^{i \theta} p^{-k / 2}, t p^{k / 2} e^{-i \theta} ; p\right)_{\infty}} d \theta \\
+\frac{(s ; s)_{\infty}}{2\left(1-t^{2}\right)(p ; p)_{\infty}} \sum_{j=0}^{k-1} \frac{\left(t^{2} ; p\right)_{j}\left(t^{2} p^{2 j}, t^{-2} p^{-2 j} ; s\right)_{\infty}}{(1 / p ; 1 / p)_{j}}
\end{gathered}
$$

Furthermore,

$$
\lim _{t \rightarrow p^{-k / 2}} G_{2}(t, p, p, s)=\frac{1}{2\left(1-p^{-k}\right)(p ; p)_{\infty}} \sum_{j=0}^{k}\left[\begin{array}{l}
k \\
j
\end{array}\right]_{p}\left(s, p^{2 j-k}, p^{k-2 j} ; s\right)_{\infty} p^{j(j-k)} .
$$

Proof. The proof of the analytic continuation formula is by induction on $k$ and is very similar to the proof of Theorem 4. In going from a value of $k$ to $k+1$ we first restrict $t$ to $p^{(1-k) / 2}<|t|<p^{-k / 2}$. We then replace the integral on $|z|=1$ by an integral on $|z|=p^{-1 / 2}$, pick up only one pole in the ring $1<|z|<p^{-1 / 2}$, namely $z=p^{-k / 2} / t$, compute the residue, then replace $z$ by $z q^{-1 / 2}$ in the integral. To establish the limiting result, let $t \rightarrow p^{-k / 2}$ in $G_{2}(t, p, p, s)$ and apply Theorem 1. The result is that the limit is

$$
\sum_{j=0}^{k} \frac{\left(p^{-k} ; p\right)_{j}\left(s, p^{2 j-k}, p^{k-2 j} ; s\right)_{\infty}}{2(p ; p)_{\infty}\left(1-p^{-k}\right)(1 / p ; 1 / p)_{j}}
$$

and the result follows.

\section{3. $q$-Hermite polynomials}

The tribasic integrals $G_{1}(t, p, q, s)$ and $G_{2}(t, p, q, s)$ of $\S 2$ are closely related to $q$-Hermite polynomials via an orthogonality relation. In this section we give the basic facts about the $q$-Hermite polynomials. We then show that evaluating a wide class of integrals, including $G_{1}(t, p, q, s)$ and $G_{2}(t, p, q, s)$ is equivalent to solving a connection coefficient problem for polynomials.

The $q$-Hermite polynomials $H_{n}(x \mid q)$ may be defined [11, p. 26] by the generating function

$$
\sum_{n=0}^{\infty} \frac{H_{n}(\cos \theta \mid q)}{(q ; q)_{n}} t^{n}=\frac{1}{\left(t e^{i \theta}, t e^{-i \theta} ; q\right)_{\infty}} .
$$

The key fact we need for these polynomials is the orthogonality relation [11 p. 188]

$$
\frac{(q ; q)_{\infty}}{2 \pi} \int_{0}^{\pi} H_{m}(\cos \theta \mid q) H_{n}(\cos \theta \mid q)\left(e^{2 i \theta}, e^{-2 i \theta} ; q\right)_{\infty} d \theta=(q ; q)_{n} \delta_{m n} .
$$


Suppose that $r_{n}(x)$ is another set of polynomials, $\operatorname{deg}\left(r_{n}\right)=n$, whose generating function is

$$
R(x, t)=\sum_{n=0}^{\infty} r_{n}(x) t^{n}
$$

To evaluate the integral

$$
I=\frac{(q ; q)_{\infty}}{2 \pi} \int_{0}^{\pi} R(\cos \theta, t)\left(e^{2 i \theta}, e^{-2 i \theta} ; q\right)_{\infty} d \theta,
$$

it is sufficient to know the connection coefficients $c_{n k}$,

$$
r_{n}(x)=\sum_{k=0}^{n} c_{n, k} H_{k}(x \mid q)
$$

The orthogonality relation (3.2) implies that

$$
I=\sum_{n=0}^{\infty} c_{n, 0} t^{n} .
$$

This is our alternate evaluation of an integral, and it will be the sum side of a Rogers-Ramanujan identity. What is required are suitable choices of the generating function $R(x, t)$ for which the constants $c_{n, 0}$ can be computed.

Two such examples for $R(x, t)$ are given by $q$-Hermite polynomials themselves,

$$
\begin{aligned}
& \sum_{n=0}^{\infty} \frac{H_{2 n}(x \mid q)}{\left(q^{2} ; q^{2}\right)_{n}} t^{n}=\frac{(-t ; q)_{\infty}}{\left(t e^{2 i \theta}, t e^{-2 i \theta} ; q^{2}\right)_{\infty}}, \\
& \sum_{n=0}^{\infty} \frac{H_{n}\left(x \mid q^{2}\right)}{(q ; q)_{n}} t^{n}=\frac{\left(q t^{2} ; q^{2}\right)_{\infty}}{\left(t e^{i \theta}, t e^{-i \theta} ; q\right)_{\infty}} .
\end{aligned}
$$

Proof of (3.5a) and (3.5b). The Askey-Wilson integral [11, p. 140]

$$
\begin{aligned}
\frac{(q ; q)_{\infty}}{2 \pi} \int_{0}^{\pi} \frac{\left(e^{2 i \theta}, e^{-2 i \theta} ; q\right)_{\infty}}{\left(a e^{i \theta}, a e^{-i \theta}, b e^{i \theta}, b e^{-i \theta}, c e^{i \theta}, c e^{-i \theta}, d e^{i \theta}, d e^{-i \theta} ; q\right)_{\infty}} d \theta \\
\quad=\frac{(a b c d ; q)_{\infty}}{(a b, a c, a d, b c, b d, c d ; q)_{\infty}},
\end{aligned}
$$

implies (3.5a) and (3.5b). To show (3.5a), expand the right-hand side in $q$-Hermite polynomials as

$$
R=\sum_{m=0}^{\infty} b_{m}(t) H_{m}(x \mid q)
$$

Then multiply $R$ by the $q$-Hermite generating function $1 /\left(s e^{i \theta}, s e^{-i \theta} ; q\right)_{\infty}$, and integrate against the $q$-Hermite weight. Then (3.2) and (3.6) with $a=\sqrt{t}, b=-\sqrt{t}$, $c=s$, and $d=0$ imply

$$
\sum_{m=0}^{\infty} b_{m}(t) s^{m}=\frac{1}{\left(t s^{2} ; q^{2}\right)_{\infty}} .
$$

The $q$-binomial theorem [11, p. 7] then implies that $b_{2 m}(t)=t^{m} /\left(q^{2} ; q^{2}\right)_{m}$ and $b_{2 m+1}(t)=0$, completing the proof of $(3.5 \mathrm{a})$. The proof of $(3.5 \mathrm{~b})$ is nearly identical. 
Besides the orthogonality relation (3.2) we will use

$$
\frac{(q ; q)_{\infty}}{2 \pi} \int_{0}^{\pi} e^{2 i n \theta}\left(e^{2 i \theta}, e^{-2 i \theta} ; q\right)_{\infty} d \theta=(-1)^{n} q^{\left(\begin{array}{c}
n \\
2
\end{array}\right)}\left(1+q^{n}\right) / 2, n=0,1, \cdots
$$

which is well known and follows from (1.1).

\section{4. $q$-HERMite CHANGE OF BASE}

In this section we consider the integral (3.3) for the generating functions (3.5a) and $(3.5 \mathrm{~b})$ on base $p$,

$$
\begin{gathered}
J_{p, q}(t)=G_{1}\left(t, p^{2}, p, q\right)=\frac{(q ; q)_{\infty}(-t ; p)_{\infty}}{2 \pi} \int_{0}^{\pi} \frac{\left(e^{2 i \theta}, e^{-2 i \theta} ; q\right)_{\infty}}{\left(t e^{2 i \theta}, t e^{-2 i \theta} ; p^{2}\right)_{\infty}} d \theta \\
H_{p, q}(t)=G_{2}\left(t, p, p^{2}, q^{2}\right)=\frac{\left(q^{2} ; q^{2}\right)_{\infty}\left(p t^{2} ; p^{2}\right)_{\infty}}{2 \pi} \int_{0}^{\pi} \frac{\left(e^{2 i \theta}, e^{-2 i \theta} ; q^{2}\right)_{\infty}}{\left(t e^{i \theta}, t e^{-i \theta} ; p\right)_{\infty}} d \theta
\end{gathered}
$$

along with the $p$-version of (3.1),

$$
I_{p, q}(t)=\frac{G_{2}\left(t, p, q^{\prime}, q\right)}{\left(p t^{2} ; q^{\prime}\right)_{\infty}}=\frac{(q ; q)_{\infty}}{2 \pi} \int_{0}^{\pi} \frac{\left(e^{2 i \theta}, e^{-2 i \theta} ; q\right)_{\infty}}{\left(t e^{i \theta}, t e^{-i \theta} ; p\right)_{\infty}} d \theta
$$

where $q^{\prime}$ is arbitrary. The bulk of this section is devoted to evaluating these integrals via the method of $\S 3$, and giving partition theory consequences of the results.

Theorems 2 and 3 give the infinite product evaluations

$$
\begin{aligned}
\lim _{t \rightarrow-1} J_{p, q}(t) & =\frac{(q,-q,-q ; q)_{\infty}}{(p,-p,-p ; p)_{\infty}} \\
\lim _{t \rightarrow p^{-1 / 2}} H_{p, q}(t) & =\frac{(-p ; p)_{\infty}\left(q^{2}, p, q^{2} / p ; q^{2}\right)_{\infty}}{(p ; p)_{\infty}} .
\end{aligned}
$$

while Theorem 3 implies

$$
\lim _{t \rightarrow p^{-1 / 2}}\left(1-p t^{2}\right) I_{p, q}(t)=\frac{(q, p, q / p ; q)_{\infty}}{(p, p ; p)_{\infty}} .
$$

For the sum sides, as in $\S 3$, let

$$
H_{n}(x \mid p)=\sum_{k=0}^{n / 2} c_{n, n-2 k}(p, q) H_{n-2 k}(x \mid q)
$$

so that

$$
\begin{gathered}
J_{p, q}(t)=\sum_{n=0}^{\infty} \frac{c_{2 n, 0}(p, q)}{\left(p^{2} ; p^{2}\right)_{n}} t^{n}, \\
H_{p, q}(t)=\sum_{n=0}^{\infty} \frac{c_{2 n, 0}\left(p^{2}, q\right)}{(p ; p)_{2 n}} t^{2 n}, \\
I_{p, q}(t)=\sum_{n=0}^{\infty} \frac{c_{2 n, 0}(p, q)}{(p ; p)_{2 n}} t^{2 n} .
\end{gathered}
$$


Thus all that remains is to find $c_{n 0}(p, q)$. However, $c_{n k}(p, q)$ is known [7], [10] (7.2)] to be given as a sum

$$
\begin{aligned}
c_{n, n-2 k}(p, q)= & \sum_{j=0}^{k}(-1)^{j} p^{k-j} q^{\left(\begin{array}{c}
j+1 \\
2
\end{array}\right)}\left[\begin{array}{c}
n-2 k+j \\
j
\end{array}\right]_{q} \\
& \times\left(\left[\begin{array}{c}
n \\
k-j
\end{array}\right]_{p}-p^{n-2 k+2 j+1}\left[\begin{array}{c}
n \\
k-j-1
\end{array}\right]_{p}\right)
\end{aligned}
$$

so that

$$
c_{2 n, 0}(p, q)=\sum_{j=-n}^{n}(-1)^{j} p^{n-j} q^{\left(\begin{array}{c}
j+1 \\
2
\end{array}\right)}\left[\begin{array}{c}
2 n \\
n-j
\end{array}\right]_{p} .
$$

We will be concerned with the values of $p$ such that the constant term $c_{2 n, 0}(p, q)$ explicitly factors. For example, it is well known that

$$
c_{2 n, 0}\left(q^{-1}, q\right)=q^{-n^{2}}\left(q^{2 n} ; q^{-1}\right)_{n}=q^{-n^{2}}(q ; q)_{2 n} /(q ; q)_{n},
$$

but other choices of $p$ may also be made (see the Appendix):

$$
\begin{aligned}
& p=q^{2}, \quad c_{2 n, 0}\left(q^{2}, q\right)=(-1)^{n} q^{n^{2}}\left(q ; q^{2}\right)_{n}, \\
& p=-q, \quad c_{2 n, 0}(-q, q)=(-q)^{n}\left(-1 ; q^{2}\right)_{n}, \\
& p=q^{1 / 2}, \quad c_{2 n, 0}\left(q^{1 / 2}, q\right)=q^{n / 2}\left(q^{1 / 2} ; q\right)_{n}, \\
& p=q^{1 / 3}, \quad c_{2 n, 0}\left(q^{1 / 3}, q\right)=q^{n / 3}\left(q^{2 n / 3} ; q^{-1 / 3}\right)_{n}, \\
& p=q^{2 / 3}, \quad c_{2 n, 0}\left(q^{2 / 3}, q\right)=q^{2 n / 3}\left(q^{1 / 3} ; q^{2 / 3}\right)_{n} .
\end{aligned}
$$

Proofs of the five evaluations (4.5a)-(4.5e) of the sum (4.4) will be given in the Appendix, $\S 9$.

For the $J_{p, q}(t)$ integral (4.1a), the evaluations (4.5) give

$$
\begin{aligned}
J_{q^{2}, q}(t) & =\sum_{n=0}^{\infty} \frac{\left(q ; q^{2}\right)_{n}}{\left(q^{4} ; q^{4}\right)_{n}} q^{n^{2}}(-t)^{n}, \\
J_{-q, q}(t) & =\sum_{n=0}^{\infty} \frac{\left(-1 ; q^{2}\right)_{n}}{\left(q^{2} ; q^{2}\right)_{n}}(-q t)^{n}=\frac{\left(q t ; q^{2}\right)_{\infty}}{\left(-q t ; q^{2}\right)_{\infty}}, \\
J_{q, q^{2}}(t) & =\sum_{n=0}^{\infty} \frac{\left(q ; q^{2}\right)_{n}}{\left(q^{2} ; q^{2}\right)_{n}}(q t)^{n}=\frac{\left(q^{2} t ; q^{2}\right)_{\infty}}{\left(q t ; q^{2}\right)_{\infty}} \\
J_{q, q^{3}}(t) & =\sum_{n=0}^{\infty} \frac{(q ; q)_{2 n}}{(q ; q)_{n}\left(q^{2} ; q^{2}\right)_{n}}(q t)^{n} \\
J_{q^{2}, q^{3}}(t) & =\sum_{n=0}^{\infty} \frac{\left(q ; q^{2}\right)_{n}}{\left(q^{4} ; q^{4}\right)_{n}}\left(q^{2} t\right)^{n} .
\end{aligned}
$$

The product evaluations for (4.6b) and (4.6c) follow from the Askey-Wilson integral (3.6). If $t=-1$, then (4.6a) factors, by a limiting case of a ${ }_{2} \phi_{1}$ summation theorem [11, (II.8)], while (4.6d) and (4.6e) give new results for $t=-1$ via (4.1a). 
Theorem 6. We have

(A) $\quad \sum_{n=0}^{\infty} \frac{\left(-q ; q^{2}\right)_{n}}{(-q ;-q)_{n}} q^{n}=\frac{\left(-q^{1},-q^{5} ; q^{6}\right)_{\infty}}{\left(q^{2}, q^{4} ; q^{6}\right)_{\infty}}=\frac{1}{\left(q^{1}, q^{4}, q^{5}, q^{7}, q^{8}, q^{11} ; q^{12}\right)_{\infty}}$

(B) $\quad \sum_{n=0}^{\infty} \frac{\left(q ; q^{2}\right)_{n}}{\left(q^{4} ; q^{4}\right)_{n}}\left(-q^{2}\right)^{n}=\frac{\left(q^{2}, q^{6}, q^{6}, q^{10} ; q^{12}\right)_{\infty}}{\left(q^{3}, q^{4}, q^{8}, q^{9} ; q^{12}\right)_{\infty}}$.

Proof. Use (4.6d), (4.6e), (4.1b) and (4.1a), and replace $q$ by $-q$ to obtain (A).

By using

$$
\frac{\left(-q ; q^{2}\right)_{n}}{(-q ;-q)_{n}} q^{n}= \begin{cases}\frac{\left(-q^{1+2 N} ; q^{2}\right)_{N}}{\left(q^{2} ; q^{2}\right)_{N}} q^{2 N} & \text { if } n=2 N \\ \frac{\left(-q^{3+2 N} ; q^{2}\right)_{N}}{\left(q^{2} ; q^{2}\right)_{N}} q^{2 N+1} & \text { if } n=2 N+1\end{cases}
$$

Theorem 6 (A) has the following integer partition interpretation, [3].

Corollary 1. Let $A(n)$ be the number of integer partitions of $n$ into parts congruent to $\pm 1, \pm 4$, or $\pm 5 \bmod 12$. Let $B(n)$ be the number of integer partitions of $n$

(1) whose odd parts are distinct and lie between the largest even part and twice the largest even part, or

(2) which have a single part of size 1, and whose other odd parts are distinct, greater than two more than the largest even part, and at most one more than twice the largest even part.

Then $A(n)=B(n)$.

In Corollary 1, the partitions for $A(10)=8$ are

$$
\text { 811, 7111, 55, 541, 511111, 4411, 4111111, 1111111111, }
$$

while those for $B(10)=8$ are

$$
10,82,641,622,442,4222,22222,5221 .
$$

For the $H_{p, q}(t)$ integral (4.1b), the evaluations (4.5) become

$$
\begin{aligned}
H_{q^{2}, q}(t) & =\sum_{n=0}^{\infty} \frac{q^{2 n^{2}}\left(-t^{2}\right)^{n}}{\left(q^{4} ; q^{4}\right)_{n}}=\left(q^{2} t^{2} ; q^{4}\right)_{\infty}, \\
H_{i q, q}(t) & =\sum_{n=0}^{\infty} \frac{\left(-1 ; q^{4}\right)_{n}}{(i q ; i q)_{2 n}}(-q t)^{2 n} \\
H_{q, q^{2}}(t) & =\sum_{n=0}^{\infty} \frac{\left(q^{2} ; q^{4}\right)_{n}}{(q ; q)_{2 n}}\left(q^{2} t^{2}\right)^{n}=\frac{\left(-q^{3} t^{2} ; q^{2}\right)_{\infty}}{\left(q^{2} t ; q^{2}\right)_{\infty}}, \\
H_{q, q^{3}}(t) & =\sum_{n=0}^{\infty} \frac{(-q ; q)_{2 n}}{\left(q^{2} ; q^{2}\right)_{n}}(q t)^{2 n} \\
H_{q^{2}, q^{3}}(t) & =\sum_{n=0}^{\infty} \frac{\left(q^{4} t^{2}\right)^{n}}{\left(q^{4} ; q^{4}\right)_{n}}=\frac{1}{\left(q^{4} t^{2} ; q^{4}\right)_{\infty}} .
\end{aligned}
$$

Again (3.6) establishes the product representations for (4.7a), (4.7c) and (4.7e). 
Theorem 7. We have

$$
\sum_{n=0}^{\infty} \frac{(-q ; q)_{2 n}}{\left(q^{2} ; q^{2}\right)_{n}} q^{n}=\frac{\left(q^{6} ; q^{6}\right)_{\infty}}{(q ; q)_{\infty}\left(q^{3} ; q^{6}\right)_{\infty}} .
$$

Proof. Use (4.2a) and (4.7d).

Using

$$
\frac{(-q ; q)_{2 n}}{\left(q^{2} ; q^{2}\right)_{n}} q^{n}=\frac{\left(-q^{1+n} ; q\right)_{n}}{(q ; q)_{n}} q^{n}
$$

we have the following corollary.

Corollary 2. Let $A(n)$ be the number of integer partitions of $n$ into parts not congruent to $0 \bmod 6$, whose parts that are congruent to $3 \bmod 6$ are colored red and blue. Let $B(n)$ be the number of integer partitions of $n$ whose parts are colored red and blue; the red parts are distinct and greater than the largest blue part and at most twice the largest blue part. Then $A(n)=B(n)$.

In Corollary 2, the partitions for $A(5)=9$ are

$$
5,41,32, \underline{3} 2,311, \underline{3} 11,221,2111,11111,
$$

where the red parts are underlined, while those for $B(5)=9$ are

$$
11111, \underline{2} 111, \underline{3} 2,221,2111,32,311,41,5 \text {. }
$$

Finally, we turn to $I_{p, q}(t)$. The evaluations for $I_{p, q}(t)$ which follow from (4.5c) and $(4.5 \mathrm{~d})$,

$$
I_{q, q^{3}}(t)=\frac{1}{\left(q t^{2} ; q\right)_{\infty}}, \quad I_{q, q^{2}}(t)=\frac{1}{\left(q t^{2} ; q\right)_{\infty}},
$$

are independently established by the the Askey-Wilson integral (2.7).

The remaining three evaluations $(4.5 \mathrm{a}),(4.5 \mathrm{~b})$ and $(4.5 \mathrm{e})$ become

$$
\begin{aligned}
I_{q^{2}, q}(t) & =\sum_{n=0}^{\infty} \frac{\left(q ; q^{2}\right)_{n}}{\left(q^{2} ; q^{2}\right)_{2 n}}(-1)^{n} q^{n^{2}} t^{2 n}, \\
I_{-q, q}(t) & =\sum_{n=0}^{\infty} \frac{\left(-1 ; q^{2}\right)_{n}}{(-q ;-q)_{2 n}}\left(-q t^{2}\right)^{n}, \\
I_{q^{2}, q^{3}}(t) & =\sum_{n=0}^{\infty} \frac{\left(q ; q^{2}\right)_{n}}{\left(q^{2} ; q^{2}\right)_{2 n}}\left(q^{2} t^{2}\right)^{n},
\end{aligned}
$$

whose (4.3a) evaluations are

$$
\begin{gathered}
\sum_{n=0}^{\infty} \frac{\left(q ; q^{2}\right)_{n}}{\left(q^{2} ; q^{2}\right)_{2 n}}(-1)^{n} q^{(n-1)^{2}-n}\left(q^{2 n-1}+q\left(\left(1-q^{4 n}\right)\left(1+q^{2 n-1}\right)\right)=0,\right. \\
\sum_{n=0}^{\infty} \frac{\left(-1 ; q^{2}\right)_{n-1}}{(-q ;-q)_{2 n}} q^{2(n-1)}\left(1-q+q^{2}+q^{2 n+1}\right)=\frac{\left(-1 ; q^{2}\right)_{\infty}}{\left(-q, q^{2} ; q^{2}\right)_{\infty}}, \\
\sum_{n=0}^{\infty} \frac{\left(q ; q^{2}\right)_{n}}{\left(q^{2} ; q^{2}\right)_{2 n}} q^{2 n-1}\left(q^{2 n+1}-1+q^{4 n}\right)=\frac{\left(q ; q^{2}\right)_{\infty}}{\left(q^{2} ; q^{2}\right)_{\infty}}
\end{gathered}
$$

One may evaluate the integral $I_{-q, q}(t)$ by other elementary means for special choices of $t$. Here we take $t=1$ and $t=q$. 
Theorem 8. We have

$$
\begin{aligned}
\sum_{n=0}^{\infty} \frac{\left(-1 ; q^{2}\right)_{n}}{(q ; q)_{2 n}} q^{n} & =\frac{\left(-q ; q^{2}\right)_{\infty}}{(q ; q)_{\infty}}\left(q^{6},-q^{2},-q^{4} ; q^{6}\right)_{\infty}, \\
\sum_{n=0}^{\infty} \frac{\left(-q^{2} ; q^{2}\right)_{n}}{(q ; q)_{2 n+1}} q^{n} & =\frac{\left(-q ; q^{2}\right)_{\infty}}{(q ; q)_{\infty}}\left(q^{6},-q^{6},-q^{6} ; q^{6}\right)_{\infty} .
\end{aligned}
$$

Proof. For (A) we evaluate

$$
\begin{aligned}
I_{-q, q}(1) & =\frac{(q ; q)_{\infty}}{2 \pi} \int_{0}^{\pi} \frac{\left(e^{2 i \theta}, e^{-2 i \theta} ; q\right)_{\infty}}{\left(e^{i \theta}, e^{-i \theta} ;-q\right)_{\infty}} d \theta \\
& =\frac{(q ; q)_{\infty}}{4 \pi} \int_{-\pi}^{\pi} \frac{\left(e^{2 i \theta}, e^{-2 i \theta}, q^{2} e^{2 i \theta}, q^{2} e^{-2 i \theta} ; q^{4}\right)_{\infty}\left(q e^{2 i \theta}, q e^{-2 i \theta} ; q^{2}\right)_{\infty}}{\left(e^{i \theta}, e^{-i \theta},-q e^{i \theta},-q e^{-i \theta} ; q^{2}\right)_{\infty}} d \theta \\
& =\frac{(q ; q)_{\infty}}{4 \pi} \int_{-\pi}^{\pi}\left(-e^{i \theta},-e^{-i \theta}, q e^{i \theta}, q e^{-i \theta}, q e^{2 i \theta}, q e^{-2 i \theta} ; q^{2}\right)_{\infty} d \theta .
\end{aligned}
$$

Using the Jacobi triple product identity (1.1) we have

$$
I_{-q, q}(1)=\frac{(q ; q)_{\infty}}{4 \pi\left(q^{2} ; q^{2}\right)_{\infty}^{3}} \sum_{m, n, j=-\infty}^{\infty}(-1)^{n+j} q^{m^{2}+m+n^{2}+j^{2}} \int_{-\pi}^{\pi} e^{i(m+n+2 j) \theta}\left(1+e^{i \theta}\right) d \theta .
$$

This integral has two terms, the first forces $n=-2 j-m$, the second forces $n=-m-2 j-1$. In the first case the power of $q$ is

$$
m^{2}+m+(m+2 j)^{2}+j^{2}=2(m+j)^{2}+3 j^{2}+m .
$$

So we can shift $m$ down by $j$ and the resulting double sum factors into two Jacobi triple products. For the second term the power of $q$ is

$$
m^{2}+m+(m+2 j+1)^{2}+j^{2}=2(m+j)^{2}+3 j^{2}+3 m+4 j+1,
$$

and again a shift of $m$ down by $j$ allows an evaluation, equal to the first term. The result is, after replacing $q$ by $-q$,

$$
I_{q,-q}(1)=\frac{\left(-q ; q^{2}\right)_{\infty}}{(q ; q)_{\infty}}\left(q^{6},-q^{2},-q^{4} ; q^{6}\right)_{\infty}
$$

For (B) we can similarly show that

$$
\begin{aligned}
I_{q,-q}(q) & =\sum_{n=0}^{\infty} \frac{\left(-1 ; q^{2}\right)_{n}}{(q ; q)_{2 n}} q^{3 n} \\
& =\frac{\left(-q ; q^{2}\right)_{\infty}}{(q ; q)_{\infty}}\left(q^{6},-q^{2},-q^{4} ; q^{6}\right)_{\infty}-q\left(q^{6},-1,-q^{6} ; q^{6}\right)_{\infty},
\end{aligned}
$$

and subtracting this from (A) gives (B).

For (4.8a) $I_{q^{2}, q}(1)$ and $I_{q^{2}, q}(q)$ may be evaluated in a similar manner using the quintuple product identity (1.2). The results are equivalent to two given by Slater [15, (117), (118)]. 
Proposition 1. We have

$$
\begin{aligned}
\sum_{n=0}^{\infty} \frac{\left(-q ; q^{2}\right)_{n}}{\left(q^{2} ; q^{2}\right)_{2 n}} q^{n^{2}} & =\frac{\left(-q^{1},-q^{5},-q^{7},-q^{9},-q^{13} ; q^{14}\right)_{\infty}}{\left(q^{2}, q^{4}, q^{10}, q^{12} ; q^{14}\right)_{\infty}}, \\
\sum_{n=0}^{\infty} \frac{\left(-q ; q^{2}\right)_{n}}{\left(q^{2} ; q^{2}\right)_{2 n}} q^{n^{2}+2 n} & =\frac{\left(-q^{3},-q^{5},-q^{7},-q^{9},-q^{11} ; q^{14}\right)_{\infty}}{\left(q^{4}, q^{6}, q^{8}, q^{10} ; q^{14}\right)_{\infty}} .
\end{aligned}
$$

It is clear that both sides of both parts of Proposition 1 have positive coefficients as power series in $q$. Here we state the integer partition version of Proposition 1A.

Corollary 3. Let $A(n)$ be the number of integer partitions of $n$ into parts congruent to $2,4,10$ or $12 \bmod 14$ and distinct parts congruent to $1,5,7,9$, or $13 \bmod 14$. Let $B(n)$ be the number of integer partitions of $n$

(1) whose odd parts are consecutive (starting with 1) and have multiplicity one or two,

(2) whose largest even part is at most two more than twice the largest odd part. Then $A(n)=B(n)$.

In Corollary 3, the partitions for $A(12)=12$ are

12, $102,921,75,741,7221,5421,52221,444,4422,42222,222222$

while those for $B(12)=12$ are

$$
\begin{aligned}
& \text { 44211, 422211, 2222211, 831, 6321, 4431, } \\
& \text { 43221, 322221, 43311, 332211, 53211, 5331. }
\end{aligned}
$$

The combinatorial version of Proposition 1B changes the parts mod 14 appropriately for $A(n)$ and adds an even part which is one larger than the largest odd part for $B(n)$.

We do not know the analogous specializations of (4.8e).

\section{A SECOND SET OF POLYNOMIALS}

In this section we carry out the program of $\S 3$ for the polynomials $s_{n}(x \mid q)$ defined by the generating function

$$
\sum_{n=0}^{\infty} s_{n}(x \mid q) \frac{t^{n}}{(q ; q)_{n}}=\frac{\left(q t^{2} ; q\right)_{\infty}}{\left(t e^{i \theta}, t e^{-i \theta} ; q\right)_{\infty}}, \quad x=\cos \theta .
$$

These polynomials were considered in [10, where it was shown that the choice of $p=q^{5}$ immediately leads to the Rogers-Ramanujan identities of modulus 5 .

The integral we are evaluating is

$$
S_{p, q}(t)=G_{2}(t, q, q, p)=\frac{\left(q t^{2} ; q\right)_{\infty}(p ; p)_{\infty}}{2 \pi} \int_{0}^{\pi} \frac{\left(e^{2 i \theta}, e^{-2 i \theta} ; p\right)_{\infty}}{\left(t e^{i \theta}, t e^{-i \theta} ; q\right)_{\infty}} d \theta .
$$

Note that Theorems 3 and 4 imply

$$
\begin{aligned}
\lim _{t \rightarrow q^{-1}} S_{p, q}(t) & =\frac{\left(p, q^{2}, p / q^{2} ; p\right)_{\infty}}{(q ; q)_{\infty}}, \\
\lim _{t \rightarrow q^{-1 / 2}} S_{p, q}(t) & =\frac{(p, q, p / q ; p)_{\infty}}{(q ; q)_{\infty}} .
\end{aligned}
$$


Let

$$
s_{n}(x \mid q)=\sum_{k=0}^{n} d_{n k}(p, q) H_{k}(x \mid p) .
$$

Only the even-degree polynomials $s_{2 n}(x \mid q)$ have a nonzero constant term. Thus

$$
S_{p, q}(t)=\sum_{n=0}^{\infty} d_{2 n, 0}(p, q) \frac{t^{2 n}}{(q ; q)_{2 n}} .
$$

The value of $d_{2 n, 0}(p, q)$ was found in [10, (7.4)] to be

$$
d_{2 n, 0}(p, q)=\sum_{j=0}^{n} \frac{(q ; q)_{2 n} q^{(n-j)(n+j+2)} p^{\left(\begin{array}{c}
j+1 \\
2
\end{array}\right)(-1)^{j}}}{(q ; q)_{2 j}(q ; q)_{n-j}\left(q^{2 j+2} ; q\right)_{n-j}} .
$$

We have nine choices of $p$ for which $d_{2 n, 0}(p, q)$ may be evaluated (see the Appendix for proofs):

One way to motivate the choices of $p$ given above is the value

$$
d_{40}(p, q)=\left(p-q^{3}\right)\left(p^{2}+p q^{3}-q^{5}-q^{7}\right),
$$

which simplifies for those choices of $p$.

The first four choices of $p$ evaluate $S_{p, q}(t)$ for all $t$, which are again equivalent to special cases of the Askey-Wilson integral,

$$
\begin{aligned}
S_{q, q}(t) & =\sum_{n=0}^{\infty} \frac{q^{\left(\begin{array}{c}
n \\
2
\end{array}\right)\left(-q t^{2}\right)^{n}}}{(q ; q)_{n}}=\left(q t^{2} ; q\right)_{\infty}, \\
S_{q^{2}, q}(t) & =\sum_{n=0}^{\infty} \frac{q^{n(n+1)}\left(-t^{2}\right)^{n}}{\left(q^{2} ; q^{2}\right)_{n}}=\left(q^{2} t^{2} ; q^{2}\right)_{\infty}, \\
S_{q^{3}, q}(t) & =1, \\
S_{q^{4}, q}(t) & =\sum_{n=0}^{\infty} \frac{q^{n(n+2)}\left(t^{2}\right)^{n}}{\left(q^{2} ; q^{2}\right)_{n}}=\left(-q^{3} t^{2} ; q^{2}\right)_{\infty} .
\end{aligned}
$$


The $S$ series corresponding to the last five choices do not have closed form sums and give new integral evaluations:

$$
\begin{aligned}
S_{q^{5}, q}(t) & =\sum_{n=0}^{\infty} \frac{q^{n^{2}+2 n} t^{2 n}}{(q ; q)_{n}} \\
S_{q^{5}, q^{2}}(t) & =\sum_{n=0}^{\infty} \frac{\left(q ; q^{2}\right)_{n}}{\left(q^{2} ; q^{2}\right)_{2 n}} q^{n(3 n+2)}\left(-t^{2}\right)^{n}, \\
S_{q^{7}, q^{2}}(t) & =\sum_{n=0}^{\infty} \frac{\left(q ; q^{2}\right)_{n}}{\left(q^{2} ; q^{2}\right)_{2 n}} q^{2 n(n+2)} t^{2 n}, \\
S_{-q^{3}, q}(t) & =\sum_{n=0}^{\infty} \frac{\left(-1 ; q^{2}\right)_{n}}{(q ; q)_{2 n}} q^{n(n+2)} t^{2 n}, \\
S_{\omega q^{3}, q}(t) & =1+(1-\omega) \sum_{n=1}^{\infty} \frac{\left(q^{3} ; q^{3}\right)_{n-1}}{(q ; q)_{2 n}(q ; q)_{n-1}} q^{n(n+2)} t^{2 n} .
\end{aligned}
$$

The choices of $t=1 / q, 1 / q^{2}$ in (5.3e), combined with (5.2a) and (5.2b), give the Rogers-Ramanujan identities of modulus 5 [3, p. 104]

$$
\begin{aligned}
& \sum_{n=0}^{\infty} \frac{q^{n^{2}}}{(q ; q)_{n}}=\frac{1}{\left(q^{1}, q^{4} ; q^{5}\right)_{\infty}}, \\
& \sum_{n=0}^{\infty} \frac{q^{n^{2}+n}}{(q ; q)_{n}}=\frac{1}{\left(q^{2}, q^{3} ; q^{5}\right)_{\infty}} .
\end{aligned}
$$

If we use (5.2a) and (5.2b) in (5.4f)-(5.4h), we find six identities, which appear in Slater's [15] list as (19), (15), (32), (33), (48), (47), respectively:

$$
\begin{aligned}
& \sum_{n=0}^{\infty} \frac{\left(-q ; q^{2}\right)_{n}}{\left(q^{2} ; q^{2}\right)_{2 n}} q^{3 n^{2}}=\frac{\left(-q^{3},-q^{5},-q^{7} ; q^{10}\right)_{\infty}}{\left(q^{4}, q^{6} ; q^{10}\right)_{\infty}}, \\
& \sum_{n=0}^{\infty} \frac{\left(-q ; q^{2}\right)_{n}}{\left(q^{2} ; q^{2}\right)_{2 n}} q^{3 n^{2}-2 n}=\frac{\left(-q^{1},-q^{5},-q^{9} ; q^{10}\right)_{\infty}}{\left(q^{2}, q^{8} ; q^{10}\right)_{\infty}}, \\
& \sum_{n=0}^{\infty} \frac{\left(-q ; q^{2}\right)_{n}}{\left(q^{2} ; q^{2}\right)_{2 n}} q^{2 n^{2}+2 n}=\frac{\left(-q^{5},-q^{7},-q^{9} ; q^{14}\right)_{\infty}}{\left(q^{4}, q^{6}, q^{8}, q^{10} ; q^{14}\right)_{\infty}}, \\
& \sum_{n=0}^{\infty} \frac{\left(-q ; q^{2}\right)_{n}}{\left(q^{2} ; q^{2}\right)_{2 n}} q^{2 n^{2}}=\frac{\left(-q^{3},-q^{7},-q^{11} ; q^{14}\right)_{\infty}}{\left(q^{2}, q^{6}, q^{8}, q^{12} ; q^{14}\right)_{\infty}}, \\
& \sum_{n=0}^{\infty} \frac{\left(-1 ; q^{2}\right)_{n}}{(q ; q)_{2 n}} q^{n^{2}+n}=\frac{\left(-q^{2},-q^{3},-q^{4} ; q^{6}\right)_{\infty}}{\left(q^{2}, q^{3}, q^{4} ; q^{6}\right)_{\infty}}, \\
& \sum_{n=0}^{\infty} \frac{\left(-1 ; q^{2}\right)_{n}}{(q ; q)_{2 n}} q^{n^{2}}=\frac{\left(-q^{1} ; q^{2}\right)_{\infty}}{\left(q ; q^{2}\right)_{\infty}} .
\end{aligned}
$$

Note that (5.4f) and (5.4g) are companion results for Proposition 1. Thus combinatorial interpretations can be stated by changing the multiplicity of the odd parts to change the power of $q$ in the sum. 
Proposition 2. Let $A(n)$ be the number of integer partitions of $n$ into parts congruent to 4 or $6 \bmod 10$ and distinct parts congruent to 3, 5, or $7 \bmod 10$. Let $B(n)$ be the number of integer partitions of $n$

(1) whose odd parts are consecutive (starting with 1) and have multiplicity three or four,

(2) whose largest even part is at most two more than twice the largest odd part. Then $A(n)=B(n)$.

Proposition 3. Let $A(n)$ be the number of integer partitions of $n$ into parts congruent to 2, 6, 8, or $12 \bmod 14$ and distinct parts congruent to 3,7 , or $11 \bmod 14$. Let $B(n)$ be the number of integer partitions of $n$

(1) whose odd parts are consecutive (starting with 1) and have multiplicity two or three,

(2) whose largest even part is at most two more than twice the largest odd part. Then $A(n)=B(n)$.

For (5.4i) we obtain the following results.

Theorem 9. We have

$$
\begin{aligned}
& 1+(1-\omega) \sum_{n=1}^{\infty} \frac{\left(q^{3} ; q^{3}\right)_{n-1}}{(q ; q)_{2 n}(q ; q)_{n-1}} q^{n(n+1)}=\frac{\left(\omega q^{3}, \omega q^{2}, q ; \omega q^{3}\right)_{\infty}}{(q ; q)_{\infty}} \\
& 1+(1-\omega) \sum_{n=1}^{\infty} \frac{\left(q^{3} ; q^{3}\right)_{n-1}}{(q ; q)_{2 n}(q ; q)_{n-1}} q^{n^{2}}=\frac{\left(\omega q^{3}, q^{2}, \omega q ; \omega q^{3}\right)_{\infty}}{(q ; q)_{\infty}}
\end{aligned}
$$

Another form of the integral representation (5.4e) is provided by the NassrallahRahman integral [11, (6.4.1)], as ${ }_{3} \phi_{2}$ on base $q^{5}$. This gives a quintic transformation which immediately becomes the first Rogers-Ramanujan identity if $t=1 / q$.

Remark 1 ([10, Theorem 7.1]). We have

$$
\sum_{n=0}^{\infty} \frac{q^{n^{2}+2 n} t^{2 n}}{(q ; q)_{n}}=\frac{\left(t^{4} q^{9}, t^{2} q^{5}, t^{4} q^{6} ; q^{5}\right)_{\infty}}{\left(t^{2} q^{3} ; q\right)_{\infty}}{ }_{3} \phi_{2}\left(\begin{array}{ccc}
t^{2} q^{2}, & t^{2} q^{3}, & t^{2} q^{5} \\
& t^{4} q^{9}, & t^{4} q^{6}
\end{array} \mid q^{5}, t^{2} q^{5}\right) .
$$

We do not know the analogous extensions of $(5.4 \mathrm{f}),(5.4 \mathrm{~g})$ and $(5.4 \mathrm{~h})$ that include the variable $t$. These should be 10th degree, 14th degree, and 12th degree transformations, and may involve multiple sums.

\section{6. $m$-VERSIONS}

The Rogers-Ramanujan identities have the natural generalization [9], 10], 12]

$$
\sum_{n=0}^{\infty} \frac{q^{n^{2}+m n}}{(q ; q)_{n}}=\frac{a_{m}(q)}{\left(q, q^{4} ; q\right)_{\infty}}+\frac{b_{m}(q)}{\left(q^{2}, q^{3} ; q\right)_{\infty}}
$$

where $a_{m}(q)$ and $b_{m}(q)$ are Laurent polynomials in $q$ which are explicitly known. We refer to (6.1) as an " $m$-version" of the Rogers-Ramanujan identities.

In this section we find $m$-versions for the identities in $\S 5$ for $m<0$ via Theorem 5 . We shall see that Theorem 5 gives a single sum for the appropriate polynomials that replace $a_{m}(q)$ and $b_{m}(q)$. In this section we will also use generating functions to find an alternative representation for these polynomials: in this multisum form the polynomials clearly have a constant sign, and positivity results are immediate. There will also be results given in this form for $m>0$. 
According to Theorem 5,

$$
\lim _{t \rightarrow q^{-m / 2}} S_{p, q}(t)=\frac{1}{2\left(1-q^{-m}\right)} \sum_{j=0}^{m}\left[\begin{array}{c}
m \\
j
\end{array}\right]_{q} \frac{\left(p, q^{2 j-m}, q^{m-2 j} ; p\right)_{\infty}}{(q ; q)_{\infty}} q^{j(j-m)},
$$

for $m=1,2, \ldots$.

For each choice of $p$ in (5.4), $S_{p, q}(t)$ is an analytic function of $t$; thus we may replace $t$ by $q^{-m / 2}$. For example, in $(5.4 \mathrm{e})$,

$$
S_{q^{5}, q}\left(q^{-m / 2}\right)=\sum_{n=0}^{\infty} \frac{q^{n^{2}+(2-m) n}}{(q ; q)_{n}} .
$$

Next we combine several of the cases in $\S 5$ into one case. Let $s$ be an odd integer that is relatively prime to a positive integer $b$. It is clear from (6.2) that $S_{q^{s}, q^{b}}\left(q^{-b m / 2}\right)$ is a linear combination of infinite products

$$
F_{i}(s, b)=\frac{\left(q^{s}, q^{i}, q^{s-i} ; q^{s}\right)_{\infty}}{\left(q^{b} ; q^{b}\right)_{\infty}}, \quad 1 \leq i \leq(s-1) / 2
$$

with rational functions of $q$ as coefficients.

Propositions 4 and 5 give two explicit forms of these rational functions.

Proposition 4. Let $s \geq 3$ be an odd positive integer relatively prime to b. Let $\beta$ be the inverse of $b \bmod s$, so that $b \beta=1+T s$. Then we have

$$
S_{q^{s}, q^{b}}\left(q^{-b m / 2}\right)=\sum_{i=1}^{(s-1) / 2} c_{i}(m, s, b) F_{i}(s, b),
$$

where

$$
\begin{aligned}
& c_{i}(m, s, b)=(-1)^{T i+1} \sum_{t}\left[\begin{array}{c}
m \\
(m+\beta i) / 2+t s
\end{array}\right]_{q^{-b}} \frac{1-q^{b(2 t s+\beta i)}}{1-q^{-b m}} q^{e}, \\
& e=-b \beta i-b \beta T i^{2}+s\left(\begin{array}{c}
T i+1 \\
2
\end{array}\right)-2 b^{2} \beta i t-b s t-2 b^{2} s t^{2} \text { for } m+\beta i \text { even, } \\
& c_{i}(m, s, b)=(-1)^{T i+1+b} \sum_{t}\left[\begin{array}{c}
m \\
(m+\beta i-s) / 2+t s
\end{array}\right]_{q^{-b}} \frac{1-q^{b((2 t-1) s+\beta i)}}{1-q^{-b m}} q^{e}, \\
& e=(-b \beta i+b s-2 t b s)((2 t-1) b+T i+1)+s\left(\begin{array}{c}
(2 t-1) b+T i+1 \\
2
\end{array}\right)
\end{aligned}
$$

for $m+\beta i$ odd.

Proof. We first consider $m+\beta i$ even so that $b(m-2 j)= \pm i \bmod s$ implies that $j=(m \mp \beta i) / 2+t s$ for some integer $t$. Upon extracting the coefficient of $F_{i}(s, b)$, we see that the choice of $+\beta i$ gives the same value as $-\beta i$, this leaves only the stated single sum and eliminates the denominator factor of 2 . The $m+\beta i$ odd case is done similarly.

Because of the factor $1-q^{-b m}$ in the denominator of Proposition 4 , it is not clear that $c_{i}(m, s, b)$ is in fact a polynomial in $q^{-b}$ with nonnegative coefficients. If we use the following elementary fact,

$$
\left[\begin{array}{c}
m \\
j
\end{array}\right]_{q} \frac{1-q^{m-2 j}}{1-q^{m}}=\left[\begin{array}{c}
m-2 \\
j
\end{array}\right]_{q}-q^{m-2 j}\left[\begin{array}{l}
m-2 \\
m-j
\end{array}\right]_{q}
$$


we see that for $m+\beta i$ even,

$$
\begin{gathered}
(-1)^{T i} q^{b \beta T i^{2}-s\left(\begin{array}{c}
T i+1 \\
2
\end{array}\right)} c_{i}(m, s, b)=\sum_{t}\left[\begin{array}{c}
m-2 \\
(m-\beta i) / 2-t s
\end{array}\right]_{q^{-b}} q^{-2 b^{2} \beta i t+b s t-2 b^{2} s t^{2}} \\
-\sum_{t}\left[\begin{array}{c}
m-2 \\
(m+\beta i) / 2-t s
\end{array}\right]_{q^{-b}} q^{-b \beta i+2 b^{2} \beta i t+b s t-2 b^{2} s t^{2}}
\end{gathered}
$$

which shows that $c_{i}(m, s, b)$ is a hook difference polynomial (see [6]).

Proposition 5. The coefficient of $F_{i}(s, b)$ is a polynomial in $1 / q$ explicitly given by the hook polynomial

$$
\begin{gathered}
\left.c_{i}(m, s, b)=(-1)^{T i} q^{-b \beta T i^{2}+s\left({ }_{2}{ }_{2}+1\right.}\right) D_{K, I}(N, M ; \alpha, \beta)\left(q^{-b}\right), \quad \text { for } m+\beta i \text { even, } \\
K=s, N=(m-\beta i) / 2, M=(m+\beta i) / 2-2, I=\beta i, \alpha=2 b-1, \beta=1 ;
\end{gathered}
$$

and for $m+\beta i$ odd,

$$
\begin{gathered}
c_{i}(m, s, b)=(-1)^{T i+b} q^{s\left(\begin{array}{c}
T i+1 \\
2
\end{array}\right)-s\left(\begin{array}{c}
b+1 \\
2
\end{array}\right)+b \beta i(b-T i)} D_{K, I}(N, M ; \alpha, \beta)\left(q^{-b}\right), \\
K=s, N=(m-\beta i+s) / 2, M=(m+\beta i-s) / 2-2, \\
I=\beta i-s, \alpha=2 b-1, \beta=1 .
\end{gathered}
$$

Next, we consider the choices of $b$ and $s$ given in $\S 5$. In these cases we shall give recurrences satisfied by the polynomials $c_{i}(m, s, b)$. Solving these recurrences gives manifestly positive expressions for the polynomials, as well as results for positive values of $m$.

The case (5.4e). In this case we have $s=5$ in Propositions 4 and 5 ,

$$
S_{q^{5}, q}\left(q^{-m / 2}\right)=\tilde{E}_{m-2}(q):=\sum_{n=0}^{\infty} \frac{q^{n(n+2-m)}}{(q ; q)_{n}}=\frac{c_{1}(m, 5,1)}{\left(q^{2}, q^{3} ; q^{5}\right)_{\infty}}+\frac{c_{2}(m, 5,1)}{\left(q^{1}, q^{4} ; q^{5}\right)_{\infty}}
$$

We now give alternative forms for the polynomials $c_{1}(m, 5,1)$ and $c_{2}(m, 5,1)$. The easily verified $q$-difference equation ,

$$
\tilde{E}_{m+1}(q)=\tilde{E}_{m}(q)+q^{-m} \tilde{E}_{m-1}(q),
$$

indicates that $c_{i}(m+2,5, q)$ also satisfy (6.5). (The reason is that $1 /\left(q, q^{4} ; q^{5}\right)_{\infty}$ and $1 /\left(q^{2}, q^{3} ; q^{5}\right)$ are linearly independent over the field of Laurent polynomials; see $\S 8$.) The initial conditions are

$$
c_{1}(1,5,1)=1, \quad c_{1}(2,5,1)=0, \quad c_{2}(1,5,1)=0, \quad c_{2}(2,5,1)=1 .
$$

Equation (6.5) is a well-studied [2] $q$-version of the Fibonacci recurrence, the solutions of which are

$$
\begin{aligned}
& c_{1}(m, 5,1)=\sum_{j=0}^{(m-3) / 2} q^{-j^{2}-j}\left[\begin{array}{c}
m-j-3 \\
j
\end{array}\right]_{q^{-1}}, \\
& c_{2}(m, 5,1)=\sum_{j=0}^{(m-2) / 2} q^{-j^{2}}\left[\begin{array}{c}
m-j-2 \\
j
\end{array}\right]_{q^{-1}} .
\end{aligned}
$$

These explicit forms may be found using generating functions. We will carry out the details in the subsequent cases. The equality of the forms (6.6) and those given by Proposition 4 are well-known polynomial identities which imply the RogersRamanujan identities. 
The case (5.4f). We set

$$
\begin{aligned}
S_{q^{5}, q^{2}}\left(q^{-m}\right) & =\tilde{F}_{m-1}(q):=\sum_{n=0}^{\infty} \frac{\left(q ; q^{2}\right)_{n}}{\left(q^{2} ; q^{2}\right)_{2 n}}(-1)^{n} q^{3 n^{2}+2 n-2 m n} \\
& =c_{1}(m, 5,2) \frac{\left(q^{5}, q^{1}, q^{4} ; q^{5}\right)_{\infty}}{\left(q^{2}, q^{2}\right)_{\infty}}+c_{2}(m, 5,2) \frac{\left(q^{5}, q^{2}, q^{3} ; q^{5}\right)_{\infty}}{\left(q^{2}, q^{2}\right)_{\infty}} .
\end{aligned}
$$

It is easy to verify that $\tilde{F}$ satisfies the recurrence relation

$$
\tilde{F}_{m+2}+q^{-1} \tilde{F}_{m+1}-\tilde{F}_{m}-q^{-1} \tilde{F}_{m-1}+q^{-1-2 m} \tilde{F}_{m-1}=0 ;
$$

hence $f_{m}=c_{i}(m+1,5,2)$ satisfy

$$
f_{m+2}+q^{-1} f_{m+1}-f_{m}-q^{-1} f_{m-1}+q^{-1-2 m} f_{m-1}=0 .
$$

The initial conditions are

$$
\begin{array}{lll}
c_{1}(1,5,2)=0, & c_{1}(2,5,2)=1, & c_{1}(3,5,2)=-q^{-1}, \\
c_{2}(1,5,2)=1, & c_{2}(2,5,2)=0, & c_{2}(3,5,2)=1 .
\end{array}
$$

Next, we explicitly solve the recurrence relation (6.8) using generating functions. Let $f_{m}$ be a solution to (6.8) and let $s=q^{-1}$ and assume for the time being that $|s|<1$. If $F(t)=\sum_{n=0}^{\infty} f_{n} t^{n}$, then $F$ satisfies

$$
\begin{aligned}
F(t) & =\frac{\phi(t)-s^{3} t^{3} F\left(s^{2} t\right)}{\left(1-t^{2}\right)(1+s t)} \\
\phi(t) & :=f_{0}(1+s t)+f_{1} t .
\end{aligned}
$$

This leads to

$$
F(t):=\sum_{n=0}^{\infty} f_{n} t^{n}=\sum_{m=0}^{\infty} \frac{(-1)^{m} t^{3 m} s^{3 m^{2}} \phi\left(t s^{2 m}\right)}{\left(-t, t,-s t ; s^{2}\right)_{m+1}} .
$$

Thus if $f_{0}=0, f_{1}=1, \phi(t)=t$, we have $c_{1}(m+1,5,2)=f_{m}$,

$$
c_{1}(n+1,5,2)=(-1)^{n-1} \sum_{3 m+2 j+k+1=n}\left[\begin{array}{c}
m+j \\
j
\end{array}\right]_{q^{-4}}\left[\begin{array}{c}
m+k \\
k
\end{array}\right]_{q^{-2}} q^{-3 m^{2}-2 m-k},
$$

while $f_{0}=1, f_{1}=0, \phi(t)=1+$ st yields

$$
c_{2}(n+1,5,2)=(-1)^{n} \sum_{3 m+2 j+k=n}\left[\begin{array}{c}
m+j \\
j
\end{array}\right]_{q^{-4}}\left[\begin{array}{c}
m+k-1 \\
k
\end{array}\right]_{q^{-2}} q^{-3 m^{2}-k} .
$$

We have two forms for the polynomials $c_{i}(n, 5,2)$, which is a polynomial identity. Somewhat surprisingly, unlike case (5.4e), a new identity, not (5.4f), results from a limit.

From Proposition 4 it is easy to see that if $Q=1 / q,|Q|<1$,

$$
\begin{aligned}
\lim _{m \rightarrow \infty} c_{i}(m, s, b)= & (-1)^{T i+1} Q^{b \beta T i^{2}-s\left(\begin{array}{c}
T i+1 \\
2
\end{array}\right)} /\left(Q^{b} ; Q^{b}\right)_{\infty} \\
& \times\left(\left(Q^{4 b^{2} s},-Q^{2 b^{2}(s+\beta i)-b s},-Q^{2 b^{2}(s-\beta i)+b s} ; Q^{4 b^{2} s}\right)_{\infty}\right. \\
& \left.-Q^{b \beta i}\left(Q^{4 b^{2} s},-Q^{2 b^{2}(s+\beta i)+b s},-Q^{2 b^{2}(s-\beta i)-b s} ; Q^{4 b^{2} s}\right)_{\infty}\right) .
\end{aligned}
$$


Evaluating this limit using the positive forms gives

$$
\begin{aligned}
& \sum_{m=0}^{\infty} \frac{q^{3 m^{2}+2 m}}{\left(q^{4} ; q^{4}\right)_{m}\left(q ; q^{2}\right)_{m+1}}+\sum_{m=0}^{\infty} \frac{(-1)^{m+1} q^{3 m^{2}+2 m}}{\left(q^{4} ; q^{4}\right)_{m}\left(-q ; q^{2}\right)_{m+1}} \\
& \quad=\frac{2 q}{\left(q^{2} ; q^{2}\right)_{\infty}}\left(\left(q^{80},-q^{54},-q^{26} ; q^{80}\right)_{\infty}-q^{6}\left(q^{80},-q^{74},-q^{6} ; q^{80}\right)_{\infty}\right), \\
& \sum_{m=0}^{\infty} \frac{q^{3 m^{2}}}{\left(q^{4} ; q^{4}\right)_{m}\left(q ; q^{2}\right)_{m}}+\sum_{m=0}^{\infty} \frac{(-1)^{m} q^{3 m^{2}}}{\left(q^{4} ; q^{4}\right)_{m}\left(-q ; q^{2}\right)_{m}} \\
& \quad=\frac{2}{\left(q^{2} ; q^{2}\right)_{\infty}}\left(\left(q^{80},-q^{38},-q^{42} ; q^{80}\right)_{\infty}-q^{2}\left(q^{80},-q^{22},-q^{58} ; q^{80}\right)_{\infty}\right) .
\end{aligned}
$$

The case $(5.4 \mathrm{~g})$. We set

$$
\begin{aligned}
S_{q^{7}, q^{2}}\left(q^{-m}\right)= & \tilde{G}_{m-2}(q):=\sum_{n=0}^{\infty} \frac{\left(q ; q^{2}\right)_{n}}{\left(q^{2} ; q^{2}\right)_{2 n}} q^{2 n^{2}+4 n-2 m n} \\
= & c_{1}(m, 7,2) \frac{\left(q^{7}, q^{1}, q^{6} ; q^{7}\right)_{\infty}}{\left(q^{2}, q^{2}\right)_{\infty}}+c_{2}(m, 7,2) \frac{\left(q^{7}, q^{2}, q^{5} ; q^{7}\right)_{\infty}}{\left(q^{2}, q^{2}\right)_{\infty}} \\
& +c_{3}(m, 7,2) \frac{\left(q^{7}, q^{3}, q^{4} ; q^{7}\right)_{\infty}}{\left(q^{2}, q^{2}\right)_{\infty}} .
\end{aligned}
$$

Analogous to the case (5.4f) one can verify that

$$
\tilde{G}_{m+2}+q^{-1} \tilde{G}_{m+1}-\left(1+q^{-2-2 m}\right) \tilde{G}_{m}-q^{-1} \tilde{G}_{m-1}=0 .
$$

Again the linear independence of $\left\{F_{1}(7,2), F_{2}(7,2), F_{3}(7,2)\right\}$ over the field of Laurent polynomials (see $\S 8)$ implies that $g_{m}=c_{i}(m+2,7,2)$ also satisfy

$$
g_{m+2}+q^{-1} g_{m+1}-\left(1+q^{-2-2 m}\right) g_{m}-q^{-1} g_{m-1}=0 .
$$

The initial conditions are

$$
\begin{aligned}
& c_{1}(1,7,2)=0, \quad c_{1}(2,7,2)=0, \quad c_{1}(3,7,2)=1, \\
& c_{2}(1,7,2)=1, \quad c_{2}(2,7,2)=0, \quad c_{2}(3,7,2)=1 \text {, } \\
& c_{3}(1,7,2)=0, \quad c_{3}(2,7,2)=1, \quad c_{3}(3,7,2)=0 .
\end{aligned}
$$

The above initial conditions show that the $c_{i}(m+2,7,2), 1 \leq i \leq 3$, forms a basis of solutions to (6.10).

Next, we solve the recurrence (6.10) using generating functions and find an explicit positive form for the polynomials $c_{i}(m+2,7,2)$.

Let $g_{m}$ be a solution to (6.10) and let $s=q^{-1}$ and assume for the time being that $|s|<1$. If $G(t)=\sum_{n=0}^{\infty} g_{n} t^{n}$, then $G$ satisfies

$$
\begin{aligned}
G(t) & =\frac{\phi(t)+s^{2} t^{2} G\left(s^{2} t\right)}{\left(1-t^{2}\right)(1+s t)}, \\
\phi(t) & :=g_{0}(1+s t)+g_{1} t++s t^{2} g_{1} .
\end{aligned}
$$

This leads to

$$
G(t):=\sum_{n=0}^{\infty} g_{n} t^{n}=\sum_{m=0}^{\infty} \frac{t^{2 m} s^{2 m^{2}} \phi\left(t s^{2 m}\right)}{\left(-t, t,-s t ; s^{2}\right)_{m+1}}
$$


In the case $g_{m}=c_{1}(m+2,7,2), \phi(t)=t$ and we find that

$$
c_{1}(n+2,7,2)=(-1)^{n-1} \sum_{2 m+2 j+k+1=n}\left[\begin{array}{c}
m+j \\
j
\end{array}\right]_{s^{4}}\left[\begin{array}{c}
m+k \\
k
\end{array}\right]_{s^{2}} s^{2 m(m+1)+k} .
$$

On the other hand, if $g_{m}=c_{2}(m+2,7,2)$, then $\phi(t)=t(1+s t)$ and we obtain

$$
c_{2}(n+2,7,2)=(-1)^{n-1} \sum_{2 m+2 j+k+1=n}\left[\begin{array}{c}
m+j \\
j
\end{array}\right]_{s^{4}}\left[\begin{array}{c}
m+k-1 \\
k
\end{array}\right]_{s^{2}} s^{2 m(m+1)+k} .
$$

Finally, $g_{m}=c_{3}(m+2,7,2)$ makes $\phi(t)=1+s t$ and we get

$$
c_{3}(n+2,7,2)=(-1)^{n} \sum_{2 m+2 j+k=n}\left[\begin{array}{c}
m+j \\
j
\end{array}\right]_{s^{4}}\left[\begin{array}{c}
m+k-1 \\
k
\end{array}\right]_{s^{2}} s^{2 m^{2}+k} .
$$

Since the expressions given for $c_{i}(n+2,7,2)$ are polynomials in $s$, we can remove the restriction $|s|<1$ and they must hold for all $s$.

This time when we get $\lim _{n \rightarrow \infty} c_{i}(n+2,7,2)$ using the two forms we obtain

$$
\begin{aligned}
& \sum_{m=0}^{\infty} \frac{q^{2 m^{2}+2 m}}{\left(q^{4} ; q^{4}\right)_{m}}\left(\frac{1}{\left(q ; q^{2}\right)_{m+1}}-\frac{1}{\left(-q ; q^{2}\right)_{m+1}}\right) \\
& \quad=\frac{2 q}{\left(q^{2} ; q^{2}\right)_{\infty}}\left(\left(q^{112},-q^{74},-q^{38} ; q^{112}\right)_{\infty}-q^{8}\left(q^{112},-q^{10},-q^{102} ; q^{112}\right)_{\infty}\right), \\
& \sum_{m=0}^{\infty} \frac{q^{2 m^{2}+2 m}}{\left(q^{4} ; q^{4}\right)_{m}}\left(\frac{1}{\left(q ; q^{2}\right)_{m+1}}+\frac{1}{\left(-q ; q^{2}\right)_{m+1}}\right) \\
& \quad=\frac{2}{\left(q^{2} ; q^{2}\right)_{\infty}}\left(\left(q^{112},-q^{46},-q^{66} ; q^{112}\right)_{\infty}-q^{6}\left(q^{112},-q^{18},-q^{94} ; q^{112}\right)_{\infty}\right), \\
& \sum_{m=0}^{\infty} \frac{q^{2 m^{2}}}{\left(q^{4} ; q^{4}\right)_{m}}\left(\frac{1}{\left(q ; q^{2}\right)_{m}}+\frac{1}{\left(-q ; q^{2}\right)_{m}}\right) \\
& \quad=\frac{2}{\left(q^{2} ; q^{2}\right)_{\infty}}\left(\left(q^{112},-q^{54},-q^{58} ; q^{112}\right)_{\infty}-q^{4}\left(q^{112},-q^{26},-q^{86} ; q^{112}\right)_{\infty}\right) .
\end{aligned}
$$

We may also explicitly find the polynomials for $c_{i}(n, 7,2)$ for $n<0$ in the same way. The results are

$$
\begin{aligned}
c_{1}(2-n, 7,2)= & (-1)^{n-2} \sum_{2 m+2 j+k+2=n}\left[\begin{array}{c}
m+j \\
j
\end{array}\right]_{q^{4}}\left[\begin{array}{c}
m+k \\
k
\end{array}\right]_{q^{2}} q^{m^{2}+2 m+1+k}, \\
c_{2}(2-n, 7,2)= & (-1)^{n-1} \sum_{2 m+2 j+k+1=n}\left[\begin{array}{c}
m+j \\
j
\end{array}\right]_{q^{4}}\left[\begin{array}{c}
m+k-1 \\
k
\end{array}\right]_{q^{2}} q^{m^{2}+k} \\
c_{3}(2-n, 7,2)= & (-1)^{n} \sum_{2 m+2 j+k=n}\left[\begin{array}{c}
m+j \\
j
\end{array}\right]_{q^{4}}\left[\begin{array}{c}
m+k-1 \\
k
\end{array}\right]_{q^{2}} q^{m^{2}-2 m+k} \\
& +(-1)^{n-1} \sum_{2 m+2 j+k+1=n}\left[\begin{array}{c}
m+j \\
j
\end{array}\right]_{q^{4}}\left[\begin{array}{c}
m+k \\
k
\end{array}\right]_{q^{2}} q^{m^{2}-1+k} .
\end{aligned}
$$

An unusual theta identity results from evaluating $\lim _{n \rightarrow \infty} c_{i}(2-n, 7,2)$ and using $\lim _{n \rightarrow-\infty} \tilde{G}_{n}(q)=0$. 
The case $(5.4 h)$ We set

$$
\tilde{H}_{m}(q)=\sum_{n=0}^{\infty} \frac{\left(-1 ; q^{2}\right)_{n}}{(q ; q)_{2 n}} q^{n(n-m)},
$$

which satisfies the three-term recurrence relation

$$
\tilde{H}_{m+2}(q)+\left[q^{-1}-q^{-1-m}\right] \tilde{H}_{m-2}=\left[1+q^{-1}+q^{-1-m}\right] \tilde{H}_{m}(q) .
$$

In this case,

$$
\tilde{H}_{m}(q)=\sum_{j=0}^{m+2}\left[\begin{array}{c}
m+2 \\
j
\end{array}\right]_{q} \frac{\left(-q^{3}, q^{2 j-m-2}, q^{m+2-2 j} ;-q^{3}\right)_{\infty}}{2\left(1-q^{-m-2}\right)(q ; q)_{\infty}} q^{-j(m+2-j)} .
$$

It is surprising that the right-hand side in the above formula changes dramatically according to whether $m$ is odd or even. It is easy to see that there are Laurent polynomials $h_{m}^{(1)}(q), h_{m}^{(2)}(q)$ and $h_{m}^{(3)}(q)$ such that

$$
\begin{aligned}
\tilde{H}_{2 m}(q)= & h_{m}^{(1)}(q) \frac{\left(-q,-q^{3},-q^{5} ; q^{6}\right)_{\infty}}{\left(q, q^{3}, q^{5} ; q^{6}\right)_{\infty}}, \\
\tilde{H}_{2 m+1}(q)= & h_{m}^{(2)}(q) \frac{\left(-1,-q^{6}, q^{3},-q^{3} ; q^{6}\right)_{\infty}}{\left(q, q^{2}, q^{4}, q^{5} ; q^{6}\right)_{\infty}} \\
& +h_{m}^{(3)}(q) \frac{\left(-q^{2},-q^{3},-q^{4} ; q^{6}\right)_{\infty}}{\left(q^{2}, q^{3}, q^{4} ; q^{6}\right)_{\infty}} .
\end{aligned}
$$

Therefore, (6.12) shows that $\left\{h_{m}^{(1)}(q)\right\}$ satisfies

$$
h_{m+1}^{(1)}(q)+q^{-1}\left[1-q^{-2 m}\right] h_{m-1}^{(1)}(q)=\left[1+q^{-1}+q^{-1-2 m}\right] h_{m}^{(1)}(q),
$$

while $\left\{h_{m}^{(2)}(q)\right\}$ and $\left\{h_{m}^{(3)}(q)\right\}$ satisfy

$$
X_{m+1}(q)+q^{-1}\left[1-q^{-2 m-1}\right] X_{m-1}(q)=\left[1+q^{-1}+q^{-2-2 m}\right] X_{m}(q) .
$$

The initial conditions are

$$
\begin{aligned}
& h_{0}^{(1)}(q)=1, \quad h_{1}^{(1)}(q)=1+2 / q, \\
& h_{-1}^{(2)}(q)=0, \quad h_{0}^{(2)}(q)=1, \quad h_{-1}^{(3)}(q)=1, \quad h_{0}^{(3)}(q)=1 .
\end{aligned}
$$

We can explicitly find $h_{n}^{(i)}(q)$ as in the previous cases; the results are

$$
\begin{gathered}
h_{n}^{(1)}(q)=\sum_{m+j+k=n}\left[\begin{array}{c}
2 m+1+j \\
j
\end{array}\right]_{q^{-1}}\left[\begin{array}{c}
m \\
k
\end{array}\right]_{q^{-2}} q^{-k^{2}-k-m^{2}}, \\
h_{n}^{(2)}(q)=\sum_{m+j+k=n}\left[\begin{array}{c}
2 m+1+j \\
j
\end{array}\right]_{q^{-1}}\left[\begin{array}{c}
m \\
k
\end{array}\right]_{q^{-2}} q^{-k^{2}-k-m^{2}-m}, \\
h_{n}^{(3)}(q)=\sum_{m+j+k=n}\left[\begin{array}{c}
2 m+j \\
j
\end{array}\right]_{q^{-1}}\left[\begin{array}{c}
m \\
k
\end{array}\right]_{q^{-2}} q^{-k^{2}-k-m^{2}-m} \\
+\sum_{m+j+k=n-1}\left[\begin{array}{c}
2 m+1+j \\
j
\end{array}\right]_{q^{-1}}\left[\begin{array}{c}
m \\
k
\end{array}\right]_{q^{-2}} q^{-k^{2}-k-m^{2}-3 m-2} .
\end{gathered}
$$


One may also appeal to orthogonal polynomials to find explicit formulas for $h_{n}^{(i)}(q)$. The three-term recurrence $(6.14 \mathrm{a})$ is a special case of that for the AlSalam-Chihara polynomials, defined by

$$
\begin{aligned}
& p_{n}\left(\cos \theta ; t_{1}, t_{2} \mid q\right)={ }_{3} \phi_{2}\left(\begin{array}{ccc}
q^{-n}, & t_{1} e^{i \theta}, & t_{1} e^{-i \theta} \\
& t_{1} t_{2}, & 0
\end{array} \mid q, q\right) \\
& =\frac{\left(t_{2} e^{-i \theta} ; q\right)_{n} t_{1}^{n} e^{i n \theta}}{\left(t_{1} t_{2} ; q\right)_{n}}{ }_{2} \phi_{1}\left(\begin{array}{c}
q^{-n}, t_{1} e^{i \theta} \\
q^{1-n} e^{i \theta} / t_{2}
\end{array} \mid q, \frac{q e^{-i \theta}}{t_{2}}\right) .
\end{aligned}
$$

Thus,

$$
h_{m}^{(1)}(q)=(-1)^{m} p_{m}\left(-\left(q^{1 / 2}+q^{-1 / 2}\right) / 2 ; q^{-1 / 2}, 0 \mid q^{-2}\right),
$$

and $(6.13 \mathrm{a})$ is

$$
\begin{aligned}
& \sum_{n=0}^{\infty} \frac{\left(-1 ; q^{2}\right)_{n}}{\left(q^{2} ; q^{2}\right)_{2 n}} q^{n(n-2 m)} \\
& =\frac{\left(-q,-q^{3},-q^{5} ; q^{6}\right)_{\infty}}{\left(q, q^{3}, q^{5} ; q^{6}\right)_{\infty}} \sum_{j=0}^{m}\left[\begin{array}{c}
m \\
j
\end{array}\right]_{q^{2}}(-1 ; q)_{2 j}(-1)^{m-j} q^{-j^{2}} .
\end{aligned}
$$

The recurrence relation $(6.14 \mathrm{~b})$ corresponds to a special case of the associated Al-Salam-Chihara polynomials [13. We shall follow the notation in Ismail and Rahman 13 . The polynomials relevant to $(6.14 \mathrm{~b})$ correspond to $b=c=d=0$ in the associated Askey-Wilson recurrence relation [13, (4.14)]

$$
\left[2 x-a r^{n+\alpha}\right] Y_{n}(x ; a \mid r)=a^{-1} Y_{n+1}(x ; a \mid r)+a\left(1-r^{n+\alpha}\right) Y_{n-1}(x ; a \mid r) .
$$

Ismail and Rahman identified two polynomial solutions to the general recurrence relation of the Askey-Wilson polynomials and in the case considered here they satisfy the initial conditions (see (4.2), (4.14), and (5.2) in [13])

$$
p_{-1}^{\alpha}(x ; a \mid r)=0, \quad p_{0}^{\alpha}(x ; a \mid r)=1, \quad q_{-1}^{\alpha}(x ; a \mid r)=1, \quad q_{0}^{\alpha}(x ; a \mid r)=1 .
$$

Now (4.15) and (8.9) of [13] give

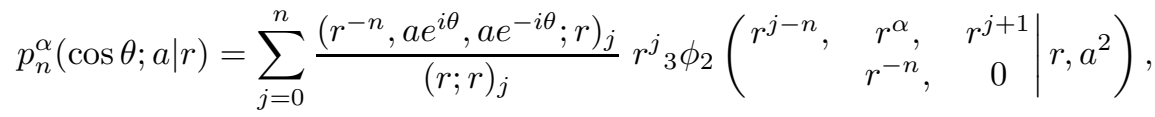

$$
\begin{aligned}
& q_{n}^{\alpha}(\cos \theta ; a \mid r)=\sum_{j=0}^{n} \frac{\left(r^{-n}, a e^{i \theta}, a e^{-i \theta} ; r\right)_{j}}{(r ; r)_{j}} r^{j}{ }_{3} \phi_{2}\left(\begin{array}{ccc}
r^{j-n}, & r^{\alpha}, & r^{j} \\
& r^{-n}, & 0
\end{array} \mid r, r a^{2}\right) .
\end{aligned}
$$

Thus,

$$
\begin{gathered}
h_{m}^{(2)}(q)=(-1)^{m} p_{m}^{1 / 2}\left(\left(q^{1 / 2}+q^{-1 / 2}\right) / 2 ;-q^{-1 / 2} \mid q^{-2}\right), \\
h_{m}^{(3)}(q)=(-1)^{m}\left[2 p_{m}^{1 / 2}\left(\left(q^{1 / 2}+q^{-1 / 2}\right) / 2 ;-q^{-1 / 2} \mid q^{-2}\right)\right. \\
\left.-q_{m}^{1 / 2}\left(\left(q^{1 / 2}+q^{-1 / 2}\right) / 2 ;-q^{-1 / 2} \mid q^{-2}\right)\right] .
\end{gathered}
$$

The case (5.4i). Set

$$
\tilde{I}_{m}(q)=1+(1-\omega) \sum_{n=1}^{\infty} \frac{\left(q^{3} ; q^{3}\right)_{n-1}}{(q ; q)_{2 n}(q ; q)_{n-1}} q^{n(n-m)} .
$$


It easily follows that $\tilde{I}_{m}$ satisfies the six-term recurrence relation

$$
\begin{aligned}
& q \tilde{I}_{m+2}(q)+\left(1+q^{-1}\right) \tilde{I}_{m-1}(q)+\tilde{I}_{m-2}(q) \\
& \quad=\tilde{I}_{m+1}(q)+\left(1+q+q^{-m}\right) \tilde{I}_{m}(q)+\left[q^{-1}-q^{-m}\right] \tilde{I}_{m-3}(q) .
\end{aligned}
$$

One can also show that $\tilde{I}_{m}$ satisfies the following inhomogeneous difference equation of order four:

$$
\begin{aligned}
\tilde{I}_{m+2} & +q^{-1}\left[1-q^{-m}\right] \tilde{I}_{m-2}-\left[1+q^{-1}+q^{-1-m}\right] \tilde{I}_{m} \\
& =q^{-1-m} \tilde{I}_{m-1}-(2+\omega) q^{-1-m} .
\end{aligned}
$$

By inspection we see that the right-hand side of (6.1) is spanned by $\left\{e_{1}, e_{2}, e_{3}, e_{4}\right\}$,

$$
e_{j}=\frac{\left(\omega q^{3}, q^{j}, q^{3-j} \omega ; \omega q^{3}\right)_{\infty}}{(q ; q)_{\infty}}, \quad 1 \leq j \leq 3, \quad e_{4}=\frac{\left(\omega q^{3}, q^{2} \omega^{2}, q \omega^{2} ; \omega q^{3}\right)_{\infty}}{(q ; q)_{\infty}} .
$$

By extending the proof of Proposition 8 , it can be shown that $\left\{e_{1}, e_{2}, e_{3}, e_{4}\right\}$ is linearly independent over the field of Laurent polynomials.

The initial conditions are

$$
\tilde{I}_{-1}(q)=e_{1}, \quad \tilde{I}_{0}(q)=e_{2}, \quad \tilde{I}_{1}=e_{1}+e_{3}, \quad \tilde{I}_{2}=\left(1+q^{-1}\right) e_{2}-\omega q^{-1} e_{4} .
$$

Furthermore, (6.1) yields

$$
q^{2} \tilde{I}_{3}=\left(1+q^{2}\right) e_{1}+\left(1+q+q^{2}\right) e_{3}-\omega e_{4} .
$$

Note that the initial conditions and the above value for $\tilde{I}_{3}$ imply

$$
e_{1}+e_{2}+\omega e_{4}=2+\omega,
$$

which can also be verified using the Jacobi triple product identity (1.1). It is easy to see that $\tilde{I}_{m}-(2+\omega) / 3$ satisfies the homogeneous recurrence relation

$$
Z_{m+2}=\left[1+q^{-1}+q^{-1-m}\right] Z_{m}+q^{-1-m} Z_{m-1}-q^{-1}\left[1-q^{-m}\right] Z_{m-2} .
$$

In view of (6.19) we set

$$
\tilde{I}_{m}=\frac{1}{3}\left[e_{1}+e_{2}+\omega e_{4}\right]+\sum_{j=1}^{4} \phi_{m}^{(j)}(q) e_{j},
$$

and find that the Laurent polynomials $\phi_{m}^{(j)}(q), 1 \leq j \leq 4$ satisfy (6.20).

As in case $(5.4 \mathrm{~g})$ we set

$$
s=1 / q, \quad Z(t)=\sum_{m=0}^{\infty} Z_{m} t^{m},
$$

and use (6.20) to derive the functional equation

$$
\begin{aligned}
Z(t) & =\frac{\phi(t)+s t^{2}\left(1+s t+s^{2} t^{2}\right) Z(s t)}{\left(1-t^{2}\right)\left(1-s t^{2}\right)} \\
\phi(t) & =Z_{0}\left[1-t^{2}(1+2 s)\right]+Z_{1} t+Z_{2} t^{2}-s(1-s) t^{3} Z_{-1} .
\end{aligned}
$$

Thus, for $|s|<1$ we establish

$$
Z(t)=\sum_{m=0}^{\infty} \frac{t^{2 m} s^{m^{2}}\left(s^{3} t^{3} ; s^{3}\right)_{m} \phi\left(t s^{m}\right)}{\left(t^{2} ; s\right)_{2 m+2}(s t ; s)_{m}}
$$

from which explicit triple sums for the Laurent polynomials $\phi_{m}^{(j)}(q), 1 \leq j \leq 4$, may be given. 


\section{Multisum Rogers-Ramanujan identities}

It is natural to ask if the $k$-fold sum of the mod $2 k+3$ Andrews-Gordon identities appears from the tribasic integrals. For example, in Theorems 3 and 4 , if $q=p$, and $s=p^{2 k+3}$, the product sides of these identities do appear. We do have double sums available for any base $p$; see (4.1b), (4.2b), (4.3b), and (5.2c) along with

$$
\begin{aligned}
& b_{n}(p, q)=p^{-n} \frac{c_{2 n, 0}(p, q)}{(p ; p)_{2 n}}=\sum_{j=0}^{n} \frac{a_{j}(p, q)}{(p ; p)_{n-j}(p ; p)_{n+j}}, \\
& a_{0}(p, q)=1, \quad a_{j}(p, q)=(-1)^{j} q^{j^{2} / 2}\left(p^{-j} q^{j / 2}+p^{j} q^{-j / 2}\right), \\
& \beta_{n}(p, q)=q^{-n^{2}-2 n} \frac{d_{2 n, 0}(p, q)}{(q ; q)_{2 n}}=\sum_{j=0}^{n} \frac{\alpha_{j}(p, q)}{(p ; p)_{n-j}(p ; p)_{n+j}} \\
& \alpha_{j}(p, q)=(-1)^{j} \frac{1-q^{2 j+1}}{1-q} q^{-j^{2}-2 j} p^{\left(\begin{array}{c}
j+1 \\
2
\end{array}\right)} .
\end{aligned}
$$

We see that $\left(a_{n}(p, q), b_{n}(p, q)\right)$ is a Bailey pair with parameters $(1, p)$, while $\left(\alpha_{n}(p, q), \beta_{n}(p, q)\right)$ is a Bailey pair with parameters $(q, q)$.

To see how the multisums arise from the double sum we use Bailey pairs. If we build a Bailey chain starting with $\left(\alpha_{n}(p, q), \beta_{n}(p, q)\right)$, then the next pair $\left(\alpha_{n}^{\prime}(p, q), \beta_{n}^{\prime}(p, q)\right)$ satisfies

$$
\alpha_{n}^{\prime}(p, q)=q^{n^{2}+n} \alpha_{n}(p, q)=\alpha_{n}\left(p q^{2}, q\right) .
$$

Thus $\beta_{n}^{\prime}\left(q^{7}, q\right)$ is a sum of the evaluable $\beta_{n}\left(q^{5}, q\right)$, and (5.2a) and (5.2b) are double sum identities for $\bmod 7$. This also works for $\bmod 2 k+3$, and for any of the nine evaluations in $\S 5$, not just (5.3e).

For the evaluations of $\S 4$ we can similarly generate multisum identities using

$$
a_{n}^{\prime}(p, q)=p^{n^{2}} a_{n}(p, q)=a_{n}\left(p^{2}, p^{2} q\right)=a_{n}\left(q, p^{2} q\right) .
$$

The change of base formulations in [8] are also available.

\section{REMARKS}

The appropriate $q \rightarrow 1$ limits of the integrals $G_{1}$ and $G_{2}$ are simple exponential integrals. For example,

$$
\lim _{q \rightarrow 1} G_{2}\left(t, q, q, q^{A}\right)=\frac{e^{-t^{2}}}{\sqrt{2 \pi}} \int_{-\infty}^{\infty} e^{-x^{2} / 2} e^{\sqrt{A} x t-t^{2} / 2} d x=e^{t^{2}(A-3) / 2} .
$$

The case $A=5$ is the motivating integral of the Introduction; see also [16].

Using the polynomials

$$
J_{n}(x \mid q)=\sum_{k=0}^{n}\left[\begin{array}{l}
n \\
k
\end{array}\right]_{q} q^{k(n-k)} e^{i(n-2 k) \theta}, \quad x=\cos \theta,
$$

whose generating function is

$$
\sum_{n=0}^{\infty} J_{n}(x \mid q) \frac{t^{n}}{(q ; q)_{n}}=\frac{\left(t^{2} ; q\right)_{\infty}}{\left(t e^{i \theta}, t e^{-i \theta} ; q\right)_{\infty}}
$$

one can evaluate an integral to conclude the following result. 
Proposition 6. We have

$$
\sum_{n=0}^{\infty} \frac{\left(-x^{2} p^{1 / 2-n / 2} ; p\right)_{n}}{(p ; p)_{n}} p^{3 n^{2} / 8} x^{-n}=\frac{\left(p^{3 / 4},-p^{3 / 8} / x,-p^{3 / 8} x ; p^{3 / 4}\right)_{\infty}}{(p ; p)_{\infty}}
$$

In fact, both the even and odd parts of this sum do sum as follows:

$$
\begin{aligned}
& \text { (B) } \sum_{n=0}^{\infty} \frac{\left(-p^{1 / 2} / x^{2},-p^{1 / 2} x^{2} ; p\right)_{n}}{(p ; p)_{2 n}} p^{n^{2}}=\frac{\left(p^{3},-p^{3 / 2} / x^{2},-p^{3 / 2} x^{2} ; p^{3}\right)_{\infty}}{(p ; p)_{\infty}} \\
& \text { (C) } \sum_{n=0}^{\infty} \frac{\left(-p^{1 / 2} / x^{2} ; p\right)_{n}\left(-p^{1 / 2} x^{2} ; p\right)_{n+1}}{(p ; p)_{2 n+1}} p^{n^{2}+n}=\frac{\left(p^{3},-p^{5 / 2} / x^{2},-p^{1 / 2} x^{2} ; p^{3}\right)_{\infty}}{(p ; p)_{\infty}} .
\end{aligned}
$$

Propositions 6(B) and 6(C) are in Ramanujan's Lost Notebook, and are special cases of Lemmas 1 and 2 in 4 . Another combination of these terms may be summed by the quintuple product identity (1.2).

Proposition 7. We have

$$
\begin{aligned}
& \sum_{n=0}^{\infty} \frac{\left(-1 / x^{3},-p^{2} x^{3} ; p^{2}\right)_{n}}{\left(p^{2} ; p^{2}\right)_{2 n}} p^{2 n^{2}}-\frac{1}{x} \sum_{n=0}^{\infty} \frac{\left(-1 / x^{3} ; p^{2}\right)_{n}\left(-p^{2} x^{3} ; p^{2}\right)_{n+1}}{\left(p^{2} ; p^{2}\right)_{2 n+1}} p^{2 n^{2}+2 n} \\
& \quad=\left(1 / x, p^{2} x ; p^{2}\right)_{\infty}\left(p^{2} x^{2}, p^{2} / x^{2} ; p^{4}\right)_{\infty}
\end{aligned}
$$

We also collect here a proposition which was used in $\S 6$.

Proposition 8. Fix a positive integer $s$, and let $G_{i}(q)=\left(q^{s}, q^{i}, q^{s-i} ; q^{s}\right)_{\infty}$ for $1 \leq i \leq\lfloor s / 2\rfloor$. Then there is no nontrivial relation

$$
\sum_{i=1}^{\lfloor s / 2\rfloor} c_{i}(q) G_{i}(q)=0
$$

where $c_{i}(q)$ is a polynomial in $q$.

Proof. By the Jacobi triple product identity we have

$$
G_{i}(q)=1+\sum_{j=1}^{\infty}(-1)^{j}\left(q^{s j^{2} / 2+(s / 2-i) j}+q^{s j^{2} / 2-(s / 2-i) j}\right) .
$$

For a fixed integer $N, G_{i}(q)$ has exactly two nonzero terms between $q^{s N^{2} / 2}$ and $q^{s(N+1)^{2} / 2}$, namely $q^{s N^{2} / 2+(s / 2-i) N}$ and $q^{s(N+1)^{2} / 2-(s / 2-i) N}$. For two different values of $i$, any pair of these terms have exponents differing by at least $N$. Thus, if $N$ is greater than the degree of any $c_{k}(q)$, the assumed relation forces all $c_{k}(q)=0$.

\section{Appendix A}

In this Appendix we evaluate the constants of $\S 4$ and $\S 5, c_{2 n, 0}(p, q)$ and $d_{2 n, 0}(p, q)$, for the choices of $p$ in (4.5) and (5.3).

Proof of (4.5). For (4.5),

$$
c_{2 n, 0}(p, q)=\sum_{j=-n}^{n}(-1)^{j} p^{n-j} q^{\left(\begin{array}{c}
j+1 \\
2
\end{array}\right)}\left[\begin{array}{c}
2 n \\
n-j
\end{array}\right]_{p},
$$


we note the alternative representation

$$
c_{2 n, 0}(p, q)=\sum_{k=0}^{2 n}(-1)^{n-k} q^{\left(\begin{array}{c}
n-k \\
2
\end{array}\right)}\left[\begin{array}{c}
2 n \\
k
\end{array}\right]_{p} .
$$

One can see that (9.1b) and (9.1a) are equivalent by computing the coefficient of $q^{\left(\begin{array}{c}j+1 \\ 2\end{array}\right)}$ in each term, $j \geq 0$.

We need Jackson's well-poised ${ }_{3} \phi_{2}$ evaluation [11, (II.15)]

$$
{ }_{3} \phi_{2}\left(\begin{array}{ccc}
r^{-2 n}, & b, & c \\
& r^{1-2 n} / b, & r^{1-2 n} / c \mid
\end{array} \mid \frac{r^{2-n}}{b c}\right)=\frac{(b, c ; r)_{n}(r, b c ; r)_{2 n}}{(r, b c ; r)_{n}(b, c ; r)_{2 n}},
$$

and the $q$-Kummer sum [11, (II.9)]

$$
{ }_{2} \phi_{1}\left(\begin{array}{c}
r^{-2 n}, b \\
r^{1-2 n} / b
\end{array} \mid r,-\frac{r}{b}\right)=\frac{\left(r^{1-2 n}, r^{2-2 n} / b^{2} ; r^{2}\right)_{n}}{\left(r^{1-2 n} / b ; r\right)_{2 n}}
$$

Rewrite (9.1a) and (9.1b) as

$$
\begin{aligned}
& c_{2 n, 0}(p, q)=(-1)^{n} \sum_{k=0}^{2 n} \frac{\left(p^{-2 n} ; p\right)_{k}}{(p ; p)_{k}} p^{k+2 n k-\left(\begin{array}{c}
k \\
2
\end{array}\right)} q^{\left(\begin{array}{c}
n-k \\
2
\end{array}\right)}, \\
& c_{2 n, 0}(p, q)=(-1)^{n} \sum_{k=0}^{2 n} \frac{\left(p^{-2 n} ; p\right)_{k}}{(p ; p)_{k}} p^{2 n k-\left(\begin{array}{c}
k \\
2
\end{array}\right)} q^{\left(\begin{array}{c}
n-k+1 \\
2
\end{array}\right)} .
\end{aligned}
$$

Proof of (4.5a, d, e). These three cases of (4.5) follow from (9.2) and (9.3).

(a) $q=p^{1 / 2}$ : use (9.3b) with $r=p^{1 / 2}, c=0, b=-p^{-n}$ in (9.2a),

(d) $q=p^{3}$ : use (9.3b) with $r=p, b, c \rightarrow \infty$ in $(9.2 \mathrm{a})$,

(e) $q=p^{3 / 2}$ : use (9.3a) with $r=p^{1 / 2}, c \rightarrow \infty, b=-p^{-n}$ in (9.2a).

Proof of (4.5c). If $q=p^{2}$ (case (4.5c)) we use the $r=p, b \rightarrow \infty$ version of (9.2b).

Proof of (4.5b). If $q=-p$ (case $(4.5 \mathrm{~b}))$ we shall use the $q$-binomial theorem,

$$
c_{2 n, 0}(p,-p)=(-1)^{n}(-p)^{\left(\begin{array}{c}
n+1 \\
2
\end{array}\right)} \sum_{k=0}^{2 n} \frac{\left(p^{-2 n} ; p\right)_{k}}{(p ; p)_{k}} p^{k(1-n)}(-1)^{n k+\left(\begin{array}{c}
k \\
2
\end{array}\right)} .
$$

First consider the case when $n$ is even in (9.4). Then $(-1)^{n k+\left(\begin{array}{l}k \\ 2\end{array}\right)}$ has sign behavior which depends only on $k$ modulo 4 ; it is ++-- . Therefore, we can evaluate the even terms in the sum by choosing $x=i$ in the $q$-binomial theorem:

$$
\sum_{k=0, k \text { even }}^{2 n} \frac{\left(p^{-2 n} ; p\right)_{k}}{(p ; p)_{k}} p^{k(1-n)}(-1)^{n k+\left(\begin{array}{c}
k \\
2
\end{array}\right)}=\frac{1}{2}\left(\left(i p^{1-n} ; p\right)_{2 n}+\left(-i p^{1-n} ; p\right)_{2 n}\right) \text {. }
$$

Similarly, the odd terms give

$$
\sum_{k=1, k \text { odd }}^{2 n} \frac{\left(p^{-2 n} ; p\right)_{k}}{(p ; p)_{k}} p^{k(1-n)}(-1)^{n k+\left(\begin{array}{c}
k \\
2
\end{array}\right)}=\frac{1}{2 i}\left(\left(i p^{1-n} ; p\right)_{2 n}-\left(-i p^{1-n} ; p\right)_{2 n}\right)
$$


thus, for $n$ even,

$$
c_{2 n, 0}(p,-p)=(-p)^{\left(\begin{array}{c}
n+1 \\
2
\end{array}\right)}\left(\frac{(1-i)}{2}\left(i p^{1-n} ; p\right)_{2 n}+\frac{(1+i)}{2}\left(-i p^{1-n} ; p\right)_{2 n}\right) .
$$

Since

$$
(1-i)\left(i p^{1-n} ; p\right)_{2 n}=(-i)^{n} p^{-\left(\begin{array}{c}
n \\
2
\end{array}\right)}\left(-1 ; p^{2}\right)_{n} \frac{1-i p^{n}}{2}
$$

a routine computation completes the proof of $(4.5 \mathrm{~b})$ for $n$ even.

For $n$ odd the sign behavior in (9.4) is +--+ ; again, we do the even and odd terms separately to find

$$
c_{2 n, 0}(p,-p)=-(-p)^{\left(\begin{array}{c}
n+1 \\
2
\end{array}\right)}\left(\frac{(1+i)}{2}\left(i p^{1-n} ; p\right)_{2 n}+\frac{(1-i)}{2}\left(-i p^{1-n} ; p\right)_{2 n}\right) .
$$

Again, it is routine to verify this case.

Proof of (5.3). For (5.3) we rewrite

$$
\begin{aligned}
d_{2 n, 0}(p, q) & =q^{n^{2}+2 n} \frac{(1-q)(q ; q)_{2 n}}{(q ; q)_{n}(q ; q)_{n+1}} \\
& \times \sum_{j=0}^{n} \frac{\left(q^{-n} ; q\right)_{j}}{\left(q^{n+2} ; q\right)_{j}} \frac{1-q^{2 j+1}}{1-q} q^{-3\left(\begin{array}{c}
j \\
2
\end{array}\right)+j(n-3)} p^{\left(\begin{array}{c}
j+1 \\
2
\end{array}\right) .}
\end{aligned}
$$

We use the very well-poised ${ }_{6} \phi_{5}$ evaluation to verify five cases of (5.2),

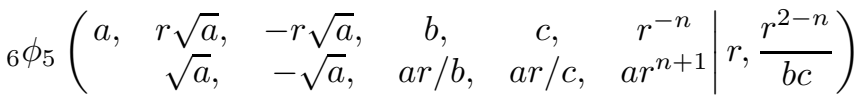

$$
\begin{aligned}
& =\frac{(a r, a r / b c ; r)_{n}}{(r, b c ; r)_{n}(a r / b, a r / c ; r)_{n}} .
\end{aligned}
$$

Proof of (5.3a) $-(5.3 \mathrm{e})$.

(a) $p=q$, use (9.7) with $r=q, a=q, b=c=0$,

(b) $p=q^{2}$, use (9.7) with $r=q, a=q, b=-q, c=0$,

(c) $p=q^{3}$, use (9.7) with $r=q, a=q, b=c=-q$,

(d) $p=q^{4}$, use (9.7) with $r=q, a=q, b=-q, c \rightarrow \infty$,

(e) $p=q^{5}$, use (9.7) with $r=q, a=q, b \rightarrow \infty, c \rightarrow \infty$.

The next two cases use the nonterminating version of Watson's transformation [11, (III.36)]



$$
\begin{aligned}
& =\frac{(a r, a r / d e, a r / d f, a r / e f ; r)_{\infty}}{(a r / d, a r / e, a r / f, a r / d e f ; r)_{\infty}}{ }_{4} \phi_{3}\left(\begin{array}{cccc}
a r / b c, & d, & e, & f \\
& a r / b, & a r / c, & d e f / a
\end{array} \mid r, r\right) \\
& +\frac{\left(a r, a r / b c, d, e, f, a^{2} r^{2} / b d e f, a^{2} r^{2} / c d e f ; r\right)_{\infty}}{\left(a r / b, a r / c, a r / d, a r / e, a r / f, a^{2} r^{2} / b c d e f, d e f / a r ; r\right)_{\infty}} \\
& \times{ }_{4} \phi_{3}\left(\begin{array}{cccc}
a r / d e, & a r / d f, & a r / e f, & a^{2} r^{2} / b c d e f \\
& a^{2} r^{2} / \text { bdef, } & a^{2} r^{2} / \text { cdef, } & a r^{2} / \operatorname{def}
\end{array} \mid r, r\right) .
\end{aligned}
$$


Proof of (5.3g). We consider case (5.3g), when $p=q^{7 / 2}$. If $r=q^{1 / 2}, a=r$, $b=i r^{3 / 2}, c=-i r^{3 / 2}, d=r^{-n}, e=-r^{-n}$, and $f \rightarrow \infty$, then we have

$$
\begin{aligned}
& c_{2 n, 0}\left(q^{7 / 2}, q\right)=q^{n^{2}+2 n} \frac{(1-q)(q ; q)_{2 n}}{(q ; q)_{n}(q ; q)_{n+1}} \\
& \times \lim _{f \rightarrow \infty} 8 \phi_{7}\left(\begin{array}{ccccccc|c}
a & r \sqrt{a}, & -r \sqrt{a}, & b, & c, & d, & e, & f \\
\sqrt{a}, & -\sqrt{a}, & a r / b, & a r / c, & a r / d, & a r / e, & a r / f
\end{array} \mid r, \frac{a^{2} r^{2}}{b c d e f}\right) .
\end{aligned}
$$

In the second term of Watson's transformation, all terms have an $f \rightarrow \infty$ limit except the pair of infinite products

$$
\frac{(f ; r)_{\infty}}{(d e f / a r ; r)_{\infty}}
$$

Let $f=\lambda r^{-m}$, and let $m \rightarrow \infty$. Then

$$
\frac{(f ; r)_{\infty}}{(d e f / a r ; r)_{\infty}}=(-1)^{m} r^{(2 n+2) m} \frac{(r / \lambda ; r)_{\infty}}{\left(-r^{2 n+3} / \lambda ; r\right)_{\infty}} \frac{(\lambda ; r)_{\infty}}{\left(-\lambda r^{-2 n-2} ; r\right)_{\infty}} .
$$

Since $r^{(2 n+2) m} \rightarrow 0$ as $m \rightarrow \infty$, the second term is zero.

What remains is the first term

$$
\frac{\left(r^{2},-r^{2 n+2} ; r\right)_{\infty}}{\left(r^{n+2},-r^{n+2} ; r\right)_{\infty}}{ }_{3} \phi_{2}\left(\begin{array}{ccc}
r^{-1}, & r^{-n}, & -r^{-n} \\
i r^{1 / 2}, & -i r^{1 / 2}
\end{array} \mid r,-r^{2 n+2}\right)
$$

This is a terminating sum of two terms, the result of which is $(5.3 \mathrm{~g})$.

Proof of (5.3f). If $p=q^{5 / 2}$, we need the same specializations of the above ${ }_{8} \phi_{7}$ but with $f \rightarrow 0$. However, it is not clear how to evaluate the $f \rightarrow 0$ limit of the second term. Thus we take a different choice of parameters, which is more delicate. Let

$$
r=q^{1 / 2}, \quad a=r, \quad e=i r^{3 / 2}, \quad d=-\epsilon i r^{3 / 2}, \quad c=-r^{-n}, \quad f=r^{-n},
$$

where $\epsilon$ is close to 1 . We take the $b \rightarrow 0$ limit of Watson's transformation for this choice. The second ${ }_{4} \phi_{3}$ is

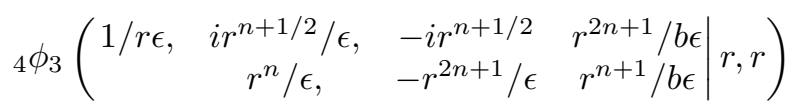

which has a limit as $b \rightarrow 0$. The infinite products multiplying this ${ }_{4} \phi_{3}$ include $(f ; r)_{\infty}=\left(r^{-n} ; r\right)_{\infty}=0$ with no zeros in the denominator; thus, the second term is again 0 .

The first term is

$$
\begin{aligned}
& \frac{\left(r^{2}, 1 / r \epsilon,-i r^{n+1 / 2} / \epsilon, i r^{n+1 / 2} ; r\right)_{\infty}}{\left(-i r^{1 / 2} / \epsilon, i r^{1 / 2}, r^{n+2}, r^{n-1} / \epsilon ; r\right)_{\infty}}
\end{aligned}
$$

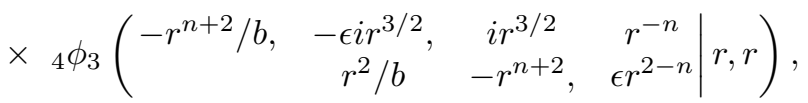

which terminates and has limit as $b \rightarrow 0$. When we let $\epsilon \rightarrow 1$, two terms of the sum survive, and the result is (5.3f).

Proof of (5.3i). If $p=\omega q^{3}$, where $\omega=e^{2 \pi i / 3}$, then we must evaluate

$$
A=\sum_{j=0}^{n} \frac{\left(q^{-n} ; q\right)_{j}}{\left(q^{n+2} ; q\right)_{j}} \frac{1-q^{2 j+1}}{1-q} q^{j n} \omega^{\left(\begin{array}{c}
j+1 \\
2
\end{array}\right) .}
$$


Since

$$
\omega^{\left({ }^{j+1}\right)}= \begin{cases}1 & \text { if } j=0 \text { or } 2 \bmod 3, \\ \omega & \text { if } j=1 \quad \bmod 3,\end{cases}
$$

the coefficient of $\omega$ in $A$ can be found by restricting the $j$-sum in (9.8) to $j=1$ mod 3. In this case we can apply (9.7) with

$$
r=q^{3}, \quad a=q^{3}, \quad b=q^{1-n}, \quad c=q^{2-n}, \quad d=q^{3-n},
$$

to evaluate the restricted sum. This gives the coefficient of $\omega$ in (5.3i).

For the coefficient of 1 , which comes from the terms in (9.8) with $j=0,2 \bmod 3$, we note by (5.3c) that the case $p=q^{3}$ sums all three terms to give 0 . Thus the coefficient of 1 is the negative of the coefficient of $\omega$; this gives the factor $(1-\omega)$ in $(5.3 \mathrm{i})$.

Proof of (5.3h). If $p=-q^{3}$, we proceed as in (5.3i). We must evaluate

$$
A=\sum_{j=0}^{n} \frac{\left(q^{-n} ; q\right)_{j}}{\left(q^{n+2} ; q\right)_{j}} \frac{1-q^{2 j+1}}{1-q} q^{j n}(-1)^{\left(\begin{array}{c}
j+1 \\
2
\end{array}\right) .}
$$

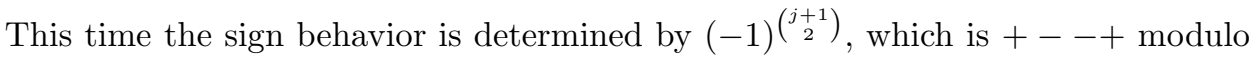
4. We shall show that the terms arising from $j=0,3$ modulo 4 sum to

$$
\left(-q^{2} ; q^{2}\right)_{n-1} \frac{(q ; q)_{n}(q ; q)_{n+1}}{(q ; q)_{2 n}(1-q)}
$$

as do the terms arising from $j=1,2$ modulo 4. Thus (9.6) shows that adding these two contributions yields $(5.3 \mathrm{~h})$.

First, we do the $j=0,3$ modulo 4 sum in (9.9). It is

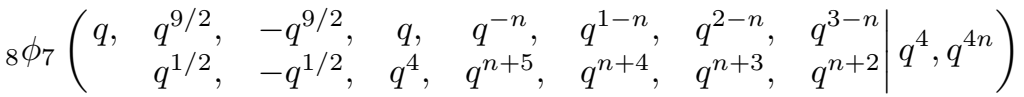

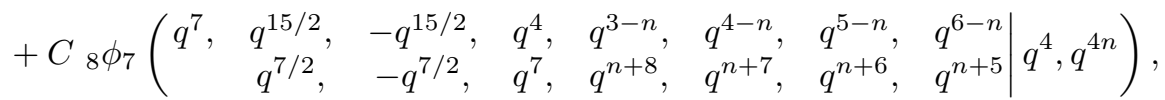

where

$$
C=\frac{\left(q^{1-n} ; q\right)_{3}}{\left(q^{n+2} ; q\right)_{3}} q^{3 n}
$$

The above sum of two very well-poised ${ }_{8} \phi_{7}$ 's can be transformed to an ${ }_{8} \psi_{8}$ using the transformation [11, (III.37)]

$$
\begin{aligned}
& \frac{(r, r / b f, r / c f, r / d f, r / e f, r f / b, r f / c, r f / d, r f / e ; r)_{\infty}}{\left(a f, r / a f, a r / f, f / a, g / f, f g, r f^{2} ; r\right)_{\infty}}
\end{aligned}
$$

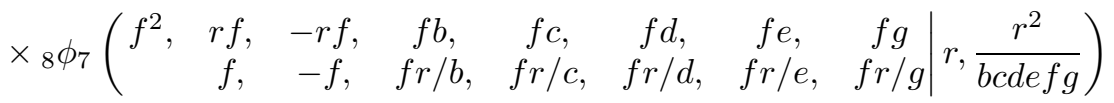

$$
\begin{aligned}
& + \text { a similar term interchanging } f \text { and } g \\
& =\frac{(a r / b, a r / c, a r / d, a r / e, r / a b, r / a d, r / a e ; r)_{\infty}}{\left(a f, a g, f / a, g / a, r a^{2}, r / a^{2} ; r\right)_{\infty}}
\end{aligned}
$$

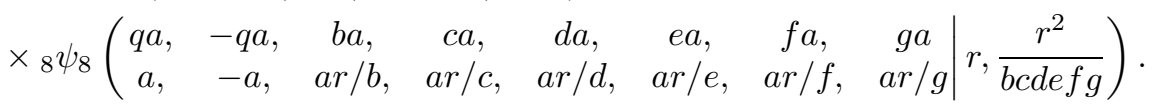


It is remarkable that the choices of $r=q^{4}, f=q^{1 / 2}, b=q^{5 / 2-n}, c=q^{-1 / 2-n}$, $d=q^{1 / 2-n}, e=q^{3 / 2-n}, g=q^{7 / 2}$, and $a=q$ make the left side of the above transformation a multiple of (9.10).

Thus we must evaluate

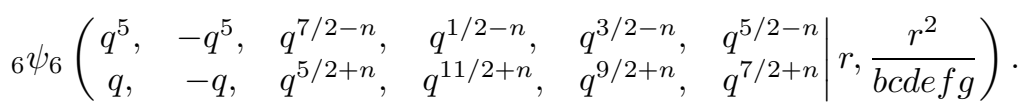

This may be summed using the very well-poised ${ }_{6} \psi_{6}$ evaluation [11, (II.33)]

$$
\begin{aligned}
& { }_{6} \psi_{6}\left(\begin{array}{cccccc|}
r \sqrt{a}, & -r \sqrt{a}, & b, & c, & d, & e \\
\sqrt{a}, & -\sqrt{a}, & a r / b, & a r / c, & a r / d, & a r / e
\end{array} \mid r, \frac{r^{2}}{b c d e}\right) \\
& =\frac{(a r, a r / b c, a r / b d, a r / b e, a r / c d, a r / c e, a r / d e, r, r / a ; r)_{\infty}}{\left(a r / c, a r / d, a r / e, r / b, r / c, r / d, r / e, r a^{2} / b c d e ; r\right)_{\infty}} .
\end{aligned}
$$

The appropriate choices in (9.11) are

$$
r=q^{4}, a=q, b=q^{7 / 2-n}, c=q^{1 / 2-n}, d=q^{3 / 2-n}, e=q^{5 / 2-n} .
$$

The details of simplifying the infinite products are onerous, but the result is that this contributes

$$
q^{n^{2}+2 n}\left(-q^{2} ; q^{2}\right)_{n-1}
$$

to $(5.3 \mathrm{~h})$.

Miraculously, the $j=1,2$ modulo 4 terms contribute an identical amount. The proof is the same, using the ${ }_{8} \psi_{8}$ transformation, this time choosing $r=q^{4}, f=q^{3 / 2}$, $b=q^{5 / 2-n}, c=q^{-1 / 2-n}, d=q^{1 / 2-n}, e=q^{3 / 2-n}, g=q^{5 / 2}, a=q$. Again a summable very well-poised ${ }_{6} \psi_{6}$ results, and the infinite products again reduce to (9.12).

\section{Appendix B}

In this appendix we verify the uniform convergence condition of Theorem 1 for the infinite products in Theorems 2, 3, 4 and 5 .

Lemma 1. If $\prod_{i=1}^{\infty} a_{i}$ and $\prod_{i=1}^{\infty} b_{i}$ converge, then

$$
\prod_{i=1}^{\infty} a_{i}-\prod_{i=1}^{\infty} b_{i}=\sum_{j=1}^{\infty}\left(\prod_{i=1}^{j} b_{i}\right)\left(a_{j}-b_{j}\right)\left(\prod_{i=j+1}^{\infty} a_{i}\right) .
$$

Proof. For any integer $J$ a telescoping sum is

$$
\prod_{i=1}^{\infty} a_{i}-\prod_{i=1}^{\infty} b_{i}=\sum_{j=1}^{J}\left(\prod_{i=1}^{j} b_{i}\right)\left(a_{j}-b_{j}\right)\left(\prod_{i=j+1}^{\infty} a_{i}\right)+b_{J}\left(\prod_{i=J+1}^{\infty} a_{i}-\prod_{i=J+1}^{\infty} b_{i}\right) .
$$

The last term approaches 0 as $J \rightarrow \infty$ because the infinite products converge. So their tails approach 1 , as do the individual terms.

For Theorem 2 note that $F\left(e^{i \theta / 2}\right)$ is uniformly bounded for $\theta \in[0,2 \pi]$. We choose

$\mathrm{SO}$

$$
a_{i}=\frac{1}{1-2 z x p^{i}+z^{2} p^{2 i}}, \quad b_{i}=\frac{1}{1+2 x p^{i}+z^{2} p^{2 i}}, \quad x=\cos \theta ;
$$

$$
a_{j}-b_{j}=\frac{p^{j}(1+z)\left(-2 x+p^{j}(1-z)\right)}{\left(1-2 z x p^{i}+z^{2} p^{2 i}\right)\left(1+2 x p^{i}+z^{2} p^{2 i}\right)} \leq M p^{j}(1+z),
$$


for a constant $M$ independent of $x$. Thus the lemma implies that

$$
\prod_{i=1}^{\infty} a_{i}-\prod_{i=1}^{\infty} b_{i} \leq M^{\prime}(1+z)
$$

A completely analogous proof works for Theorems 3, 4, and 5 .

\section{ACKNOWLEDGMENTS}

This research was partially supported by NSF grants DMS 99-70865 and DMS 99-70627. Part of this work was done while the authors were visiting the Liu Bie Ju Center of Mathematical Sciences of City University of Hong Kong and they gratefully acknowledge the generous financial support and the hospitality of the City University of Hong Kong.

\section{REFERENCES}

1. L. Ahlfors, Complex analysis, 3rd ed., McGraw-Hill, New York, 1978. MR 80c:30001

2. G. Andrews, On identities implying the Rogers-Ramanujan identities, Houston J. Math. 2 (1976), 289-298. MR 53:13148

3. 1976 original. MR 99c:11126]

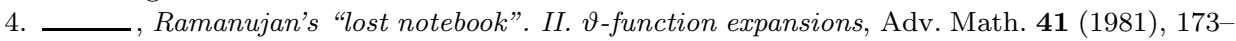
185. MR 83m:10034b

5. G. Andrews, R. Askey, and R. Roy, Special functions, Cambridge University Press, Cambridge, 1999. MR 2000g:33001

6. G. Andrews, R. Baxter, D. Bressoud, W. Burge, P. Forrester, and G. Viennot, Partitions with prescribed hook differences, European. J. Comb. 8 (1987), 341-350. MR 88g:05014

7. D. Bressoud, On partitions, orthogonal polynomials and the expansion of certain infinite products, Proc. London Math. Soc. 42 (1981), 478-500. MR 82e:05021

8. D. Bressoud, M. E. H. Ismail, and D. Stanton, Change of base in Bailey pairs, Ramanujan J. (2001), 435-453. MR 2002a:33023

9. L. Carlitz, Some formulas related to the Rogers-Ramanujan identities, Ann. Mat. Pura Appl. (4) 47 (1959), 243-251. MR 22:1704

10. K. Garrett, M. E. H. Ismail, and D. Stanton, Variants of the Rogers-Ramanujan identities, Adv. Appl. Math. 23 (1999), 274-299. MR 2000i:33028

11. G. Gasper and M. Rahman, Basic hypergeometric series, Cambridge University Press, Cambridge, 1990. MR 91d:33034

12. M. E. H. Ismail, H. Prodinger, and D. Stanton, Schur's determinants and partition theorems, Séminaire Lotharingien de Combinatoire 44 (2000), Art. B44a, 10 pp. (electronic). MR 2001d:05010

13. M. E. H. Ismail and M. Rahman, The associated Askey-Wilson polynomials, Trans. Amer. Math. Soc. 328 (1991), 201-237. MR 92c:33019]

14. I. Schur, Ein beitrag zur additiven zahlentheorie und zur theorie der kettenbrüche, S.-B Preuss. Akad. Wiss. Phys.-Math. Kl. 54 (1917), 302-321.

15. L. Slater, Further identities of the Rogers-Ramanujan type, Proc. London Math. Soc. (2) 54 (1952), 147-167. MR 14:138e

16. D. Stanton, Gaussian integrals and the Rogers-Ramanujan identities, Symbolic Computation, Number Theory, Special Functions, Physics, and Combinatorics (F. Garvan and M. E. H. Ismail, eds.), Kluwer, Dordrecht, 2001, pp. 255-265. MR 2003d:33035

Department of Mathematics, University of Central Florida, Orlando, Florida 32816

School of Mathematics, University of Minnesota, Minneapolis, Minnesota 55455 